\title{
REDUCTIONS AND EXACT SOLUTIONS OF SOME NONLINEAR PARTIAL DIFFERENTIAL EQUATIONS UNDER FOUR TYPES OF GENERALIZED CONDITIONAL SYMMETRIES
}

\author{
CHANGZHENG QU'
}

(Received 13 May 1997; revised 3 March 1998)

\begin{abstract}
The generalized conditional symmetry method is applied to study the reduction to finitedimensional dynamical systems and construction of exact solutions for certain types of nonlinear partial differential equations which have many physically significant applications in physics and related sciences. The exact solutions of the resulting equations are derived via the compatibility of the generalized conditional symmetries and the considered equations, which reduces to solving some systems of ordinary differential equations. For some unsolvable systems of ordinary differential equations, the dynamical behavior and qualitative properties are also considered. To illustrate that the approach has wide application, the exact solutions of a number of nonlinear partial differential equations are also given. The method used in this paper also provides a symmetry group interpretation to some known results in the literature which cannot be obtained by the nonclassical symmetry method due to Bluman and Cole.
\end{abstract}

\section{Introduction}

The reduction to finite-dimensional dynamical systems and construction of exact solutions are two important problems in the study of nonlinear partial differential equations (PDEs). The most effective and universal method used is the classical symmetry method pioneered by Lie [17]. But there exist very important equations that admit poor Lie symmetries. Various generalizations of the classical method have been developed, which include the method of partial invariant solutions due to Ovsiannikov [24], the nonclassical symmetry method due to Bluman and Cole [5], the direct method of Clarkson and Kruskal [6], the side conditional constraint approach of Olver and Rosenau [23] and the iteration of nonclassical symmetry method of Nucci [22]. Those generalizations have been successfully applied to construct exact solutions

\footnotetext{
${ }^{1}$ Department of Mathematics, Northwest University, Xi' an, 710069, P. R. China

(C) Australian Mathematical Society 1999, Serial-fee code 0334-2700/99
} 
of and present symmetry group classifications for a considerable number of nonlinear PDEs. Recently Fokas and Liu $[9,10]$ introduced a concept of generalized conditional symmetry (GCS) and have applied it to construct some physically interesting exact solutions $[9,10,25,26]$ of some nonlinear PDEs. The method can be thought of as a generalization of the nonclassical method.

An important class of nonlinear diffusion equations

$$
u_{t}=g\left(u, u_{x}\right) u_{x x}+f\left(u, u_{x}\right)
$$

has a wide range of applications in physics and related sciences, for example, in biophysics [20], solid state physics [21], plasma physics [29] and hydrodynamics [11]. The symmetry group reductions of (1), including the classical and the nonclassical symmetry reductions, has been studied by many authors $[3,4,7,8,30,32]$. There are some examples of (1) $[12,13,16]$ of special ansatz which reduce the equations considered to systems of ordinary differential equations (ODEs). The generalized conditional symmetry reduction of (1) was considered in [26] under ansatz on GCSs, which ensures the reduction of (1) to systems of ODEs in the cases of power law and exponential diffusivities.

The other three classes of important nonlinear PDEs, that is, the nonlinear hyperbolic equations of second order

$$
u_{t t}=g\left(u, u_{x}\right) u_{x x}+f\left(u, u_{x}\right),
$$

the generalized Kuramoto-Sivashinsky equations [19]

$$
u_{t}=-u_{x x x x}+g\left(u, u_{x}\right) u_{x x}+f\left(u, u_{x}\right),
$$

and the nonlinear elliptic equations of fourth order

$$
u_{x x x x}+2 u_{x x y y}+u_{y y y y}=g\left(u, u_{x}\right) u_{x x}+f\left(u, u_{x}\right),
$$

also have many physically significant applications in wave motion, nonlinear mechanics and physics. The symmetry group properties and classification of (2) have been discussed by several authors $[2,15]$. Ibragimov et al. [15] used a preliminary group classification technique to (2). The point Lie symmetry groups of equation (4) with $f=g=0$ is considered in $[1,27]$.

In the generalization of the nonclassical symmetry method due to Bluman and Cole [5], Olver and Rosenau [23] show that in order to determine a group-invariant solution of a given PDE, one can use any group of infinitesimal transformations. However, in general, given any group of infinitesimal transformations and any $\mathrm{PDE}(\mathrm{s})$, there will be no solutions invariant under the group and so the question becomes how to determine a priori whether a given group will classify the large class of nonlinear PDEs and 
give meaningful symmetry reductions to the resulting equations. This problem will be considered in this paper.

It is known $[12,13,16,32]$ that a large number of $1+1$ dimensional nonlinear PDE(s) possess exact solutions

$$
u(t, x)=[\alpha(t)+\beta(t) \cos (a x)+\gamma(t) \sin (a x)]^{1 / m}
$$

or

$$
u(t, x)=[\alpha(t)+\beta(t) \cosh (a x)+\gamma(t) \sinh (a x)]^{1 / m},
$$

where $a$ is a constant and $m$ is an arbitrary integer, or polynomial-type solutions of a spatial variable. A common feature of these three types of solution is that the equations considered are invariant under GCSs

$$
\sigma=u_{x x x} \pm u_{x}
$$

or

$$
\sigma=\frac{\partial^{n} u}{\partial x^{n}}, \quad n \in Z^{+}
$$

which will be described in the sequel.

This paper is devoted to discussing the reduction and construction of exact solutions of some nonlinear PDEs, including equations (1)-(4) and other important nonlinear PDEs with the generalized conditional symmetries (7)-(8). In Section 2, we give a brief discussion of the GCS method. In Section 3, we determine the admissible forms of equations (1)-(4) under the GCSs (7)-(8). The exact solutions of nonlinear PDEs (1)-(4) and the reductions to systems of ODEs are discussed in Section 4 and Appendix A. Section 5 contains a number of further examples which illustrate the wide application of the approach. The dynamical behavior and qualitative properties of some unsolvable systems of ODEs are investigated in Section 6. Section 7 is a discussion of our results.

\section{The GCS of nonlinear PDEs}

Let $K(t, u)$ denote a function which depends in a differentiable manner on $u, u_{x}$, $u_{x x}, \ldots$ and $t$. The function $\sigma(t, x, u)$ is a symmetry of the equation

$$
u_{t}=K(t, u),
$$


if and only if

$$
\frac{\partial \sigma}{\partial t}=[K, \sigma]
$$

where $[K, \sigma]=K^{\prime} \sigma-\sigma^{\prime} K$ and primes denote the Gâteaux derivative. The concept of conditional symmetry was introduced in [5] under the name of nonclassical symmetry. The GCS is a generalization of conditional symmetry. The function $\sigma(t, x, u)$ is a GCS of (9) if

$$
\frac{\partial \sigma}{\partial t}=[K, \sigma]+A(t, x, u, \sigma), \quad A(t, x, u, 0)=0,
$$

where $K(t, u)$ and $\sigma(t, x, u)$ are differentiable functions of $t, x$ and $u, u_{x}, u_{x x}, \ldots$, and $A(t, x, u, \sigma)$ a differentiable function of $t, x, u, u_{x}, u_{x x}, \ldots$ and $\sigma, \sigma_{x}, \sigma_{x x}, \ldots$ From (10), if $\sigma$ is explicitly independent of time $t$ we see that (9) admits a GCS $\sigma$ if and only if

$$
\left.\sigma^{\prime} K\right|_{\sigma=0}=0
$$

This method prevides an algorithm for constructing exact solutions of nonlinear PDEs.

\section{The admissible forms of (1)-(4) under GCSs (7)-(8)}

In this section, we determine the admissible forms of (1)-(4) under GCSs (7)-(8). The method used here is the GCS method from Section 2. If $m \neq 1$ and $a \neq 1$ in (5) and (6), by performing the transformations

$$
x^{*}=a x, \quad t^{*}=b t, \quad y^{*}=c y, \quad u^{*}=u^{m},
$$

(1)-(4) can be reduced to the same types of equations, so that, without loss of generality, we can set $a=m=1$. We consider four cases with respect to (7)-(8).

3.1. Under $\sigma_{1}=u_{x x x}+u_{x}$. From Section 2, we see that (1) admits the GCS $\sigma_{1}$ if and only if

$$
\begin{aligned}
\left.\sigma_{1}^{\prime}\left(K_{1}\right)\right|_{\sigma_{1}=0}= & g_{222} u_{x x}^{4}+\left(f_{222}+3 g_{221} u_{x}+3 g_{12}\right) u_{x x}^{3} \\
& +3\left(g_{112} u_{x}^{2}+g_{11} u_{x}-2 g_{22} u_{x}-g_{2}+f_{122} u_{x}+f_{12}\right) u_{x x}^{2} \\
& +\left(g_{111} u_{x}^{3}-9 g_{12} u_{x}^{2}-6 g_{1} u_{x}+3 f_{112} u_{x}^{2}+3 f_{11} u_{x}-3 f_{22} u_{x}\right) u_{x x} \\
& +\left(f_{111}-3 g_{11}\right) u_{x}^{3}+3\left(g_{2}-f_{12}\right) u_{x}^{2}=0
\end{aligned}
$$


where $K_{1}=g\left(u, u_{x}\right) u_{x x}+f\left(u, u_{x}\right)$ and $f_{1}$ and $f_{2}$ denote the partial derivative with respect to $u$ and $u_{x}$ throughout the paper. Equating to zero the coefficients of each polynomial term of $u_{x x}$ implies

$$
\begin{aligned}
& g_{222}=0, \quad f_{222}+3 g_{122} u_{x}+3 g_{12}=0, \\
& g_{112} u_{x}^{2}+g_{11} u_{x}-2 g_{22} u_{x}-g_{2}+f_{122} u_{x}+f_{12}=0, \\
& g_{111} u_{x}^{2}-9 g_{12} u_{x}-6 g_{1}+3 f_{112} u_{x}+3 f_{11}-3 f_{22}=0, \\
& f_{111} u_{x}+3 g_{2}-3 g_{11} u_{x}-3 f_{12}=0 .
\end{aligned}
$$

The general solution of (15) can be easily obtained as

$$
\begin{aligned}
g\left(u, u_{x}\right)= & \left(c_{1} u+c_{2}\right) u_{x}^{2}+\left(c_{3} u+c_{4}\right) u_{x}+c_{1} u^{3}+\left(3 c_{2}-2 c_{6}\right) u^{2}+c_{8} u+c_{9}, \\
f\left(u, u_{x}\right)= & -\frac{c_{1}}{2} u_{x}^{4}-\frac{c_{3}}{2} u_{x}^{3}+\left(c_{6} u+c_{7}\right) u_{x}^{2}+\left(\frac{c_{3}}{2} u^{2}+c_{4} u+c_{5}\right) u_{x}+\frac{1}{2} c_{1} u^{4} \\
& +\left(2 c_{2}-c_{6}\right) u^{3}+\left(c_{8}+c_{7}\right) u^{2}+c_{10} u+c_{11}
\end{aligned}
$$

where as hereafter, $c_{i}, i=1,2, \ldots$, are arbitrary constants. This includes the wellknown equation (6) of reference [13] as a special case.

In the same way, we can prove that if (2)-(4) admit the GCS $\sigma, g\left(u, u_{x}\right)$ and $f\left(u, u_{x}\right)$ in (2)-(4) must take the form (16).

3.2. Under $\sigma_{2}=u_{x x x}-u_{x}$. Analogously to case (3.1), (1) admits GCS $\sigma_{2}$ if and only if

$$
\begin{aligned}
\left.\sigma_{2}^{\prime}\left(K_{1}\right)\right|_{\sigma_{2}=0}= & g_{222} u_{x x}^{4}+\left(f_{222}+3 g_{122} u_{x}+3 g_{12}\right) u_{x x}^{3} \\
& +3\left[g_{112} u_{x}^{2}+\left(g_{11}+2 g_{22}+f_{122}\right) u_{x}+g_{2}\right] u_{x x}^{2} \\
& +\left[g_{111} u_{x}^{2}+\left(9 g_{12}+3 f_{112}\right) u_{x}+3\left(2 g_{1}+f_{11}+f_{22}\right)\right] u_{x} u_{x x} \\
& +\left[\left(f_{111}+3 g_{11}\right) u_{x}+3\left(g_{2}+f_{12}\right)\right] u_{x}^{2}=0
\end{aligned}
$$

Equating to zero the coefficients of each polynomial term of $u_{x x}$ yields $f$ and $g$, which satisfy the overdetermined system of ODEs

$$
\begin{aligned}
& g_{222}=0, \quad f_{222}+3 g_{122} u_{x}+3 g_{12}=0, \\
& 2 g_{112} u_{x}^{2}+\left(g_{11}+2 g_{22}+f_{122}\right) u_{x}+g_{2}=0, \\
& g_{111} u_{x}^{2}+3\left(3 g_{12}+f_{112}\right) u_{x}+6 g_{1}+3 f_{11}+3 f_{22}=0, \\
& \left(f_{111}+3 g_{11}\right) u_{x}+3 g_{2}+3 f_{12}=0 .
\end{aligned}
$$

We the obtain general solution of $(18)$ as

$$
g\left(u, u_{x}\right)=\left(c_{1} u+c_{2}\right) u_{x}^{2}+\left(c_{3} u+c_{4}\right) u_{x}-c_{1} u^{3}-\left(3 c_{2}+2 c_{6}\right) u^{2}+c_{8} u+c_{9},
$$




$$
\begin{aligned}
f\left(u, u_{x}\right)= & -\frac{c_{1}}{2} u_{x}^{4}-\frac{1}{2} c_{3} u_{x}^{2}+\left(c_{6} u+c_{7}\right) u_{x}^{2}+\left(-\frac{c_{3}}{2} u^{2}-c_{4} u+c_{5}\right) u_{x}+\frac{1}{2} c_{1} u^{4} \\
& +\left(2 c_{2}+c_{6}\right) u^{3}-\left(c_{8}+c_{7}\right) u^{2}+c_{10} u+c_{11}
\end{aligned}
$$

From (12) it is easily shown that $\sigma_{2}$ also is a GCS of (2)-(4) with (19).

3.3. Under $\sigma_{3}=u_{x x x x}$. Equation (1) admits the GCS $\sigma_{3}$ implying

$$
\begin{aligned}
\left.\sigma_{3}^{\prime}\left(K_{1}\right)\right|_{\sigma_{3}=0}= & 15 g_{22} u_{x x} u_{x x x}^{3}+\left(4 g_{1}+12 g_{12} u_{x}+3 f_{22}\right) u_{x x x}^{2} \\
& +10 g_{222} u_{x x}^{3} u_{x x x}+\left(24 g_{122} u_{x}+22 g_{12}+6 f_{222}\right) u_{x x}^{2} u_{x x x} \\
& +\left(18 g_{112} u_{x}^{2}+16 g_{11} u_{x}+12 f_{122} u_{x}+10 f_{12}\right) u_{x x} u_{x x x} \\
& +\left(4 g_{111} u_{x}^{3}+6 f_{112} u_{x}^{2}+4 f_{11} u_{x}\right) u_{x x x}+g_{1111} u_{x x}^{5} \\
& +\left(f_{1111}+4 g_{1222} u_{x}+6 g_{122}\right) u_{x x}^{4} \\
& +\left(6 g_{1122} u_{x}^{2}+\left(12 g_{112}+4 f_{1222}\right) u_{x}+3 g_{11}+6 f_{122}\right) u_{x x}^{3} \\
& +\left(4 g_{1112} u_{x}^{3}+6\left(g_{111}+f_{1122}\right) u_{x}^{2}+12 f_{112} u_{x}+3 f_{11}\right) u_{x x}^{2} \\
& +\left(g_{1111} u_{x}^{2}+4 f_{1112} u_{x}+6 f_{111}\right) u_{x}^{2} u_{x x}+f_{1111} u_{x}^{4}=0,
\end{aligned}
$$

which in turn implies $g$ and $f$ satisfy the system of ODEs

$$
\begin{aligned}
& g_{22}=0, \quad f_{2222}=0, \quad f_{1111}=0, \quad g_{1111}=0, \\
& 4 g_{1}+12 g_{12} u_{x}+3 f_{22}=0, \quad 11 g_{12}+3 f_{222}=0, \\
& 9 g_{112} u_{x}^{2}+8 g_{11} u_{x}+6 f_{122} u_{x}+5 f_{12}=0, \\
& 2 g_{111} u_{x}^{2}+3 f_{112} u_{x}+2 f_{11}=0, \\
& \left(12 g_{112}+4 f_{1222}\right) u_{x}+3 g_{11}+6 f_{1222}=0, \\
& 4 g_{1112} u_{x}^{3}+\left(6 g_{111}+6 f_{1122}\right) u_{x}^{2}+12 f_{112} u_{x}+3 f_{11}=0 \\
& g_{1111} u_{x}^{2}+4 f_{1112} u_{x}+6 f_{111}=0 .
\end{aligned}
$$

The general solution of (21) is given by

$$
\begin{aligned}
& g\left(u, u_{x}\right)=c_{1} u_{x}+c_{2} u+c_{3}, \\
& f\left(u, u_{x}\right)=-\frac{2}{3} c_{2} u_{x}^{2}+c_{5} u_{x}+c_{4} u+c_{6},
\end{aligned}
$$

which includes equation (1.6) with $\alpha=-2 / 3, N=1$ in [16]. Investigating the above proof again, we find that if (2)-(4) admit the GCS $\sigma_{3}, g$ and $f$ must possess the form (22).

3.4. Under $\sigma_{4}=u_{x x x}$. Equation (1) admits the GCS $\sigma_{4}$ giving rise to

$$
\left.\sigma_{4}^{\prime}\left(K_{1}\right)\right|_{\sigma_{4}=0}=g_{222} u_{x x}^{4}+\left(f_{222}+3 g_{12}+3 g_{122} u_{x}\right) u_{x x}^{3}
$$




$$
\begin{aligned}
& +3\left[g_{112} u_{x}^{2}+\left(g_{11}+f_{122}\right) u_{x}+f_{12}\right] u_{x x}^{2} \\
& +\left(g_{111} u_{x}^{2}+3 f_{112} u_{x}+3 f_{11}\right) u_{x} u_{x x}+f_{111} u_{x}^{3}=0 .
\end{aligned}
$$

Equating to zero of the coefficients of each polynomial term of $u_{x x}$ implies

$$
\begin{aligned}
& g_{222}=0, \quad f_{111}=0, \quad f_{222}+3 g_{12}+3 g_{122} u_{x}=0, \\
& g_{112} u_{x}^{2}+\left(g_{11}+f_{122}\right) u_{x}+f_{12}=0, \\
& g_{111} u_{x}^{2}+3 f_{112} u_{x}+3 f_{11}=0,
\end{aligned}
$$

with general solution

$$
\begin{aligned}
& g\left(u, u_{x}\right)=\left(c_{1} u+c_{2}\right) u_{x}^{2}+\left(c_{3} u+c_{4}\right) u_{x}-2 c_{6} u^{2}+c_{8} u+c_{9}, \\
& f\left(u, u_{x}\right)=-\frac{c_{1}}{2} u_{x}^{4}-\frac{c_{3}}{2} u_{x}^{3}+\left(c_{6} u+c_{7}\right) u_{x}^{2}+c_{5} u_{x}+c_{10} u+c_{11} .
\end{aligned}
$$

It is easy to show that (2)-(4) with (25) also admit the GCS $\sigma_{4}$.

\section{Exact solutions of (1)}

In this section we consider reductions of (1) to systems of ODEs to construct their exact solutions by using the compatibility of $\sigma=0$ and the equations considered. The procedure is first to solve the ODE $\sigma=0$ to derive $u$ as a function of $x$ with $x$-independent integration constants and then to substitute those solutions into the governing equations to obtain the time-evolution of those constants. Those systems of ODEs play an important role in discussing the asymptotic behaviour of the solutions, such as blow-up phenomena. Though the general solutions are difficult to derive, some exact solutions are obtained in special cases.

To obtain the exact solutions of (1), we distinguish four cases in terms of its GCSs (7)-(8).

4.1. Under $\sigma_{1}=u_{x x x}+u_{x}$. In this case, $g$ and $f$ are constrained by (16). Solving $\sigma_{1}=0$, we have

$$
u(t, x)=\alpha(t)+\beta(t) \cos x+\gamma(t) \sin x .
$$

The substitution of (26) into (1) with (16) implies that $\alpha, \beta$ and $\gamma$ satisfy the system of ODEs

$$
\alpha_{t}=L_{1}(\alpha, \beta, \gamma), \quad \beta_{t}=L_{2}(\alpha, \beta, \gamma), \quad \gamma_{t}=L_{3}(\alpha, \beta, \gamma),
$$

where $L_{1}, L_{2}$ and $L_{3}$ are given by

$$
L_{1}=\frac{1}{2} c_{1}\left[\alpha^{4}-\left(\beta^{2}+\gamma^{2}\right)^{2}\right]+2 c_{2} \alpha^{3}+c_{6} \alpha\left(\beta^{2}+\gamma^{2}-\alpha^{2}\right)
$$




$$
\begin{aligned}
& +c_{7}\left(\alpha^{2}+\beta^{2}+\gamma^{2}\right)+c_{8} \alpha^{2}+c_{10} \alpha+c_{11}, \\
L_{2}= & \left(c_{6} \beta-\frac{1}{2} c_{3} \gamma-c_{1} \alpha \beta\right)\left(\beta^{2}+\gamma^{2}-\alpha^{2}\right)+c_{2} \beta\left(3 \alpha^{2}-\beta^{2}-\gamma^{2}\right) \\
& +c_{4} \alpha \gamma+\left(2 c_{7}+c_{8}\right) \alpha \beta+\left(c_{10}-c_{9}\right) \beta+c_{5} \gamma, \\
L_{3}= & \left(c_{6} \gamma+\frac{1}{2} c_{3} \beta-c_{1} \alpha \gamma\right)\left(\beta^{2}+\gamma^{2}-\alpha^{2}\right)+c_{2} \gamma\left(3 \alpha^{2}-\beta^{2}-\gamma^{2}\right) \\
& -c_{4} \alpha \beta+\left(2 c_{7}+c_{8}\right) \alpha \gamma+\left(c_{10}-c_{9}\right) \gamma-c_{5} \beta .
\end{aligned}
$$

Even though the general solution of (27)-(28) for arbitrary $c_{i}$ is unknown, we can obtain some exact solutions of this system in the following special cases.

4.1.1. $c_{i}=0, i \neq 1$. Two exact solutions are admitted. First

(i)

$$
\begin{gathered}
\alpha= \pm \sqrt{\left(1+D_{1}^{2}\right) \beta^{2}+D_{2} \beta}, \quad \gamma=D_{1} \beta \\
-\frac{1}{3} \bar{\beta}^{\frac{3}{2}}+\left(1+D_{1}^{2}\right) \bar{\beta}^{\frac{1}{2}}=\frac{1}{2} c_{1} D_{2}^{3}\left(t-t_{0}\right), \quad D_{2} \neq 0
\end{gathered}
$$

with $\bar{\beta}=\frac{1}{\beta}+\frac{1+D_{1}^{2}}{2 D_{2}}$. As subsequently, unless otherwise stated, $D_{i}, i=1,2, \ldots$, denote arbitrary constants introduced into computations and $t_{0}$ is an arbitrary positive constant. Secondly

(ii)

$$
\alpha= \pm \sqrt{1+D_{1}^{2}} D_{2}, \quad \beta=D_{2}, \quad \gamma=D_{1} D_{2}
$$

which combined with (26) gives the steady-state solution of (1) with (16) and $c_{i}=0$, $i \neq 1$.

4.1.2. $c_{i}=0, i \neq 2,6$. Three subcases arise.

(i) $c_{2}=0$. In this subcase, the solution is given by

$$
\alpha=D_{2} \beta, \quad \gamma=D_{1} \beta, \quad \beta=\left[2 c_{6}\left(D_{2}^{2}-D_{1}^{2}-1\right)\left(t-t_{0}\right)\right]^{-\frac{1}{2}}
$$

which blows up at finite time $t_{0}$.

(ii) $c_{6}=c_{2} \neq 0$. The exact solution of (27)-(28) is implicitly given by

$$
\begin{gathered}
\alpha= \pm \sqrt{\left(1+D_{1}^{2}\right) \beta^{2}+D_{2} \beta}, \quad \gamma=D_{1} \beta \\
\frac{D_{2}}{\beta}+\left(1+D_{1}^{2}\right) \ln \frac{\beta}{D_{2}+\left(1+D_{1}^{2}\right) \beta}=-2 D_{2}^{2} c_{2}\left(t-t_{0}\right), \quad D_{2} \neq 0
\end{gathered}
$$


(iii) $c_{6}=2 c_{2} \neq 0$. The implicit exact solution is

$$
\begin{aligned}
& \beta= \pm \frac{\sqrt{\alpha^{2}-D_{2} \alpha}}{1+D_{1}^{2}}, \quad \gamma=D_{1} \beta \\
& \frac{D_{2}}{\alpha}-\ln \frac{\alpha}{\alpha-D_{2}}=D_{2}^{2} c_{6}\left(t-t_{0}\right) .
\end{aligned}
$$

4.1.3. $c_{i}=0, i \neq 4,7,8$. There are also three subcases.

(i) $c_{4}=0, c_{8}+2 c_{7} \neq 0$. Two possibilities arise.

(i.A) $c_{7}=0$.

$$
\alpha=-\frac{1}{c_{8}\left(t-t_{0}\right)}, \quad \beta=D_{1} \alpha, \quad \gamma=D_{2} \alpha .
$$

which combined with (26) gives a separable solution of (1).

(i.B) $c_{7} \neq 0$. The system (27)-(28) has the implicit exact solution

$$
\begin{gathered}
\alpha= \pm \sqrt{\left(1+D_{1}^{2}\right) \beta^{2}+D_{2} \beta^{\frac{2 c_{7}+2 c_{8}}{c_{8}+2 \sigma_{7}}}}, \quad \gamma=D_{1} \beta, \\
\int_{\beta_{0}}^{\beta} \beta^{-1}\left[\left(1+D_{1}^{2}\right) \beta^{2}+D_{2} \beta^{\frac{2 c_{8}+2 c_{8}}{\delta_{8}+2 c_{7}}}\right]^{-\frac{1}{2}} d \beta=\left(c_{8}+2 c_{7}\right)\left(t-t_{0}\right),
\end{gathered}
$$

where $\beta_{0}$ is a constant, provided the integral exists.

(ii) $c_{4} \neq 0, c_{8}+2 c_{7}=0$. In this subcase,

$$
\begin{aligned}
& \alpha=D_{1} \operatorname{coth}\left[c_{7} D_{1}\left(t-t_{0}\right)\right], \\
& \beta=D_{1} \sin \left\{c_{4} D_{1} \ln \sinh \left[c_{7} D_{1}\left(t-t_{0}\right)\right]+D_{2}\right\}, \\
& \gamma=D_{1} \cos \left\{c_{4} D_{1} \ln \sinh \left[c_{7} D_{1}\left(t-t_{0}\right)\right]+D_{2}\right\} .
\end{aligned}
$$

This form of solution is new and blows up at $t=t_{0}$ and

$$
T_{k}=t_{0}+\frac{1}{c_{7} D_{1}} \sinh ^{-1} \exp \left\{\frac{(2 k+1) \pi-2 D_{2}}{2 c_{4} D_{1}}\right\} .
$$

(iii) $c_{8}+2 c_{7} \neq 0, c_{4} \neq 0$. The general solution of system (27)-(28) in this subcase is

$$
\alpha=\frac{1}{c_{4}} h_{t}, \quad \beta=p \sin h, \quad \gamma=p \cos h,
$$

where $p$ and $h$ are given implicitly by

$$
\int_{p_{0}}^{p} s^{-2}\left[\left(c_{8}+2 c_{7}\right)^{2}+D_{2} s^{-\frac{2 c_{7}}{c_{8}+27}}\right]^{-\frac{1}{2}} d s=t-t_{0}, \quad h=\frac{c_{4}}{c_{8}+2 c_{7}} \ln p+D_{1} .
$$


If $c_{8}=-3 c_{7}$, we get an explicit solution

$$
\begin{aligned}
& \alpha=c_{7}^{5}\left(t-t_{0}\right)\left[c_{7}^{4}\left(t-t_{0}\right)^{2}-D_{2}\right]^{-2}, \\
& \beta=c_{7}\left[c_{7}^{4}\left(t-t_{0}\right)^{2}-D_{2}\right]^{-\frac{1}{2}} \sin \left[-\frac{c_{4}}{c_{7}} \ln \left[c_{7}\left(c_{7}^{4}\left(t-t_{0}\right)^{2}-D_{2}\right)^{-\frac{1}{2}}+D_{1}\right],\right. \\
& \gamma=c_{7}\left[c_{7}^{4}\left(t-t_{0}\right)^{2}-D_{2}\right]^{-\frac{1}{2}} \cos \left[-\frac{c_{4}}{c_{7}} \ln \left[c_{7}\left(c_{7}^{4}\left(t-t_{0}\right)^{2}-D_{2}\right)^{-\frac{1}{2}}+D_{1}\right] .\right.
\end{aligned}
$$

4.1.4. $c_{1}=c_{5}=c_{6}=c_{7}=0$. In this subcase

$$
\begin{aligned}
& \beta=q \sin \left[\int_{0}^{t}\left(\frac{c_{3}}{2}\left(\alpha^{2}-q\right)+c_{4} \alpha\right) d t+D_{2}\right], \\
& \gamma=q \cos \left[\int_{0}^{t}\left(\frac{c_{3}}{2}\left(\alpha^{2}-q\right)+c_{4} \alpha\right) d t+D_{2}\right],
\end{aligned}
$$

where

$$
q=\frac{e^{S}}{D_{2}+2 c_{2} \int_{0}^{t} e^{s} d t}, \quad S=2 \int_{0}^{t}\left(3 c_{2} \alpha^{2}+c_{8} \alpha+c_{10}-c_{9}\right) d t
$$

and $\alpha$ solves

$$
\alpha_{t}=2 c_{2} \alpha^{3}+c_{8} \alpha^{2}+c_{10} \alpha+c_{11} .
$$

The general solution of $(40)$ is given in Appendix B.

4.2. Under $\sigma_{2}=u_{x x x}-u_{x}$. Here $g$ and $f$ take the form (19) and $\sigma_{2}=0$ leads to

$$
u(t, x)=\alpha(t)+\beta(t) \cosh x+\gamma(t) \sinh x,
$$

which is compatible with (1) and requires that $\alpha, \beta$ and $\gamma$ satisfy the system of ODEs

$$
\alpha_{t}=L_{4}(\alpha, \beta, \gamma), \quad \beta_{t}=L_{5}(\alpha, \beta, \gamma), \quad \gamma_{t}=L_{6}(\alpha, \beta, \gamma),
$$

where $L_{4}, L_{5}$ and $L_{6}$ are given by

$$
\begin{aligned}
L_{4}= & \frac{1}{2} c_{1}\left[\alpha^{4}-\left(\beta^{2}-\gamma^{2}\right)^{2}\right]+c_{6} \alpha\left(\alpha^{2}-\beta^{2}+\gamma^{2}\right)+c_{7}\left(\gamma^{2}-\alpha^{2}-\beta^{2}\right) \\
& +2 c_{2} \alpha^{3}-c_{8} \alpha^{2}+c_{10} \alpha+c_{11}, \\
L_{5}= & \left(c_{6} \beta+c_{1} \alpha \beta-\frac{1}{2} c_{3} \gamma\right)\left(\alpha^{2}-\beta^{2}+\gamma^{2}\right)+c_{2} \beta\left(3 \alpha^{2}-\beta^{2}+\gamma^{2}\right) \\
& -c_{4} \alpha \gamma-\left(2 c_{7}+c_{8}\right) \alpha \gamma+\left(c_{9}+c_{10}\right) \beta+c_{5} \gamma, \\
L_{6}= & \left(c_{6} \gamma+c_{1} \alpha \gamma-\frac{1}{2} c_{3} \beta\right)\left(\alpha^{2}-\beta^{2}+\gamma^{2}\right)+c_{2} \gamma\left(3 \alpha^{2}+\gamma^{2}-\beta^{2}\right) \\
& -c_{4} \alpha \beta-\left(2 c_{7}+c_{8}\right) \alpha \gamma+\left(c_{9}+c_{10}\right) \gamma+c_{5} \beta .
\end{aligned}
$$

We also consider several cases. 
4.2.1. $c_{i}$ arbitrary. In this case, system (42)-(43) has the exact solution

$$
\begin{gathered}
\beta=D_{1} \exp \left[\int_{\alpha_{0}}^{\alpha} \frac{c_{1} z^{3}+\left(3 c_{2}+c_{6}-\frac{1}{2} c_{3}\right) z^{2}-\left(c_{4}+c_{8}+2 c_{7}\right) z+c_{9}+c_{10}}{\frac{1}{2} c_{1} z^{4}+\left(2 c_{2}+c_{6}\right) z^{3}-\left(c_{7}+c_{8}\right) z^{2}+c_{10} z+c_{11}} d z\right] \\
\gamma=\beta
\end{gathered}
$$

where $\alpha_{0}$ is a constant provided the integral exists and $\alpha$ solves

$$
\alpha_{t}=\frac{1}{2} c_{1} \alpha^{4}+\left(c_{6}+2 c_{2}\right) \alpha^{3}-\left(c_{7}+c_{8}\right) \alpha^{2}+c_{10} \alpha+c_{11}
$$

The general solution of (45) is given in Appendix C.

4.2.2. $c_{1}=c_{6}=c_{7}=0$. In this case $\alpha$ fulfills

$$
\alpha_{t}=2 c_{2} \alpha^{3}-c_{8} \alpha^{2}+c_{10} \alpha+c_{11}
$$

The general solution of (46) is given in Appendix B.

Setting

$$
P_{1}=\beta / \gamma, \quad H_{1}=\beta^{2}-\gamma^{2},
$$

$P_{1}$ and $H_{1}$ satisfy the system

$$
\begin{aligned}
P_{1 t} & =\left[-\frac{1}{2} c_{3}\left(\alpha^{2}-H_{1}^{2}\right)+c_{4} \alpha\right]\left(P_{1}^{2}-1\right), \\
H_{1 t} & =-2 c_{2} H_{1}^{2}+2\left[3 c_{2}^{2}-\left(c_{8}+2 c_{7}\right) \alpha+c_{9}+c_{10}\right] H_{1},
\end{aligned}
$$

with implicit exact solution

$$
\begin{aligned}
P_{1} & =\frac{1+D_{1} \exp \left[c_{3}\left(H_{1}^{2}-\alpha^{2}\right)+2 c_{4} \alpha\right]}{1-D_{1} \exp \left[c_{3}\left(H_{1}^{2}-\alpha^{2}\right)+2 c_{4} \alpha\right]} \\
H_{1} & =\frac{e^{\left(c_{10}+2 c_{9}\right) t}\left(2 c_{2} \alpha^{3}-c_{8} \alpha^{2}+c_{10} \alpha+c_{11}\right)}{2 c_{2} \alpha e^{\left(c_{10}+2 c_{9}\right) t}-2 c_{2}\left(c_{10}+2 c_{9}\right) \int_{0}^{t} \alpha e^{\left(c_{10}+2 c_{9}\right) t} d t+D_{2}}
\end{aligned}
$$

Hence from (47) we have

$$
\beta= \pm P_{1} \sqrt{\frac{H_{1}}{P_{1}^{2}-1}}, \quad \gamma= \pm \sqrt{\frac{H_{1}}{P_{1}^{2}-1}}
$$


4.2.3. $c_{i}=0, i \neq 1$. From the system (42)-(43), we find that

$$
\alpha= \pm\left[\left(1-D_{1}^{2}\right) \beta^{2}-D_{2} \beta\right]^{\frac{1}{2}}, \quad \gamma=D_{1} \beta .
$$

To determine $\beta$, there are two possibilities.

(i) $D_{2} \neq 0$. Substituting (51) into the second equation of (42) and solving gives

$$
\left(1-D_{1}^{2}-\frac{D_{2}}{\beta}\right)^{3 / 2}-3\left(1-D_{1}^{2}\right)\left(1-D_{1}^{2}-\frac{D_{2}}{\beta}\right)^{1 / 2}=\frac{3}{2} c_{1} D_{1}\left(t-t_{0}\right) \text {. }
$$

(ii) $D_{2}=0$. In this subcase, the solution of (42)-(43) is given by

$$
\alpha= \pm\left(1-D_{1}^{2}\right)^{\frac{1}{2}} D_{3}, \quad \beta=D_{3}, \quad \gamma=D_{1} D_{3},
$$

which gives a steady-state solution of (1) with (19).

4.2.4. $\quad c_{i}=0, i \neq 2,6$. Analogously to case 4.1.2, we obtain the exact solution of (42)-(43) as

(i) for $c_{2}=0$ :

$$
\alpha=D_{2} \beta, \quad \gamma=D_{1} \beta, \quad \beta=\left[-2 c_{6}\left(D_{2}^{2}+D_{1}^{2}-1\right)\left(t-t_{0}\right)\right]^{-\frac{1}{2}}
$$

(ii) for $c_{2}=-c_{6} \neq 0$ :

$$
\begin{gathered}
\alpha= \pm\left[D_{2} \beta-\left(D_{1}^{2}-1\right) \beta^{2}\right]^{\frac{1}{2}}, \quad \gamma=D_{1} \beta, \\
\frac{D_{2}}{\beta}-\left(D_{1}^{2}-1\right) \ln \frac{\beta}{D_{2}-\left(D_{1}^{2}-1\right) \beta}=-2 c_{2} D_{2}^{2}\left(t-t_{0}\right) .
\end{gathered}
$$

(iii) for $c_{6}=-2 c_{2} \neq 0$ :

$$
\begin{aligned}
& \gamma=D_{1} \beta, \quad \beta= \pm\left[\frac{\alpha^{2}-D_{2} \alpha}{1-D_{1}^{2}}\right]^{\frac{1}{2}}, \\
& \ln \frac{\alpha-D_{2}}{\alpha}-\frac{D_{2}}{\alpha}=2 c_{2} D_{2}^{2}\left(t-t_{0}\right) .
\end{aligned}
$$

4.2.5. $c_{i}=0, i \neq 4,7,8$. We distinguish three subcases.

(i) $c_{4}=0, c_{7}, c_{8}+2 c_{7} \neq 0$. From the last two equation of (42), we find that

$$
\gamma=D_{1} \beta \text {. }
$$

Substituting (57) into the first two equations of (42)-(43), we obtain

$$
\begin{gathered}
\alpha=-\left[\left(1-D_{1}^{2}\right) \beta^{2}+D_{4} \beta^{\frac{2 c_{8}+2 \eta}{c_{8}+2 \eta}}\right]^{\frac{1}{2}} \\
\int_{\beta_{0}}^{\beta} \frac{1}{\beta}\left[\left(1-D_{1}^{2}\right) \beta^{2}+D_{4} \beta^{\frac{2 c_{8}+2 c_{8}}{c_{8}+2 c^{2}}}\right]^{-\frac{1}{2}} d \beta=\left(c_{8}+2 c_{7}\right)\left(t-t_{0}\right) .
\end{gathered}
$$

When $c_{8}=-c_{7}$, we can obtain explicit solutions for $\beta$ 
(i.A) $1-D_{1}^{2}>0, D_{4}>0$ :

$$
\beta=\frac{2 \sqrt{D}_{4} e^{-c_{7} \sqrt{D_{4}\left(t-t_{0}\right)}}}{\sqrt{1-D_{1}^{2}}\left(e^{-2 c_{7} \sqrt{D}_{4}\left(t-t_{0}\right)}+1\right)} .
$$

(i.B) $1-D_{1}^{2}<0, D_{4}>0$ :

$$
\beta=\frac{2 \sqrt{D}_{4} e^{-c_{7} \sqrt{D}_{4}\left(t-t_{0}\right)}}{\sqrt{D_{1}^{2}-1}\left(e^{-2 c_{7} \sqrt{D}_{4}\left(t-t_{0}\right)}+1\right)} .
$$

(i.C) $1-D_{1}^{2}>0, D_{4}<0$ :

$$
\beta=\frac{\sqrt{-D_{4}}}{\sqrt{1-D_{1}^{2}}} \csc \left[c_{7} \sqrt{-D_{4}}\left(t-t_{0}\right)\right]
$$

which blows up at points $T_{k}=t_{0}-k \pi /\left(c_{7} \sqrt{-D_{4}}\right)$.

(i.D) $1-D_{1}^{2}>0, D_{4}=0$ :

$$
\beta=\frac{1}{c_{7} \sqrt{1-D_{1}^{2}}}\left(t-t_{0}\right)^{-1}
$$

(ii) $c_{4}=c_{8}+2 c_{7}=0$. It is easy to see that

$$
\beta=D_{1}, \quad \gamma=D_{2}
$$

and $\alpha$ is given as follows.

(ii.A) $D_{2}^{2}-D_{1}^{2}>0$ :

$$
\alpha=\sqrt{D_{2}^{2}-D_{1}^{2}} \tan \left[c_{7}\left(t-t_{0}\right)\right]
$$

which blows up at $T_{k}=t_{0}+\pi(2 k+1) /\left(2 c_{7}\right)$.

(ii.B) $D_{2}^{2}-D_{1}^{2}<0$ :

$$
\alpha=\sqrt{D_{1}^{2}-D_{2}^{2}} \operatorname{coth}\left[c_{7} \sqrt{D_{1}^{2}-D_{2}^{2}}\left(t-t_{0}\right)\right]
$$

(ii.C) $D_{1}^{2}=D_{2}^{2}$ :

$$
\alpha=-\frac{1}{c_{7}\left(t-t_{0}\right)} .
$$

(iii) $c_{4} \neq 0, c_{8}+2 c_{7} \neq 0$. We consider two special subcases. 
(iii.A) $c_{8}+2 c_{7}+c_{4}=0$. An exact solution to (42)-(43) is given by

$$
\alpha=-\sqrt{-\frac{1}{D_{1}} \phi+\frac{D_{2}}{4 c_{4}^{2}} \phi^{1+\frac{7}{c_{4}}}}, \quad \beta=\frac{1}{D_{1}}-\gamma, \quad \gamma=\frac{\phi}{2}+\frac{1}{2 D_{1}}, \quad D_{1} \neq 0,
$$

where $\phi$ is determined implicitly by

$$
\int_{\phi_{0}}^{\phi}\left[-\frac{4 c_{4}^{2}}{D_{1}} \phi^{3}+D_{2} \phi^{3+\frac{q_{7}}{c_{4}}}\right]^{-\frac{1}{2}} d \phi=t-t_{0}
$$

(iii.B) $c_{8}+2 c_{7}-c_{4}=0$. An exact solution to (42)-(43) is given by

$$
\alpha=-\sqrt{-\frac{1}{D_{1}} \psi+\frac{D_{2}}{4 c_{4}^{2}} \psi^{1-\frac{q}{c_{4}}}}, \quad \beta=\frac{1}{D_{1}}+\gamma, \quad \gamma=\frac{\psi}{2}-\frac{1}{2 D_{1}}, \quad D_{1} \neq 0,
$$

where $\psi$ is determined implicitly by

$$
\int_{\psi_{0}}^{\psi}\left[-\frac{4 c_{4}^{2}}{D_{1}} \psi+D_{2} \psi^{3-\frac{\gamma}{c_{4}}}\right]^{-\frac{1}{2}} d \psi=t-t_{0}
$$

4.3. Under $\sigma_{3}=u_{x x x x}$. In this subcase, $g$ and $f$ are given by (22). Integrating $\sigma_{3}=0$, we obtain the following ansatz for the function $u(t, x)$ :

$$
u(t, x)=\alpha(t)+\beta(t) x+\gamma(t) x^{2}+\delta(t) x^{3} .
$$

Inserting this into (1) with (22) shows that $\alpha, \beta, \gamma$ and $\delta$ satisfy the system of ODEs

$$
\begin{array}{cl}
\alpha_{t}=L_{7}(\alpha, \beta, \gamma, \delta), & \beta_{t}=L_{8}(\alpha, \beta, \gamma, \delta), \\
\gamma_{t}=L_{9}(\alpha, \beta, \gamma, \delta), & \delta_{t}=18 c_{1} \delta^{2}+c_{4} \delta,
\end{array}
$$

where $L_{7}, L_{8}$ and $L_{9}$ are given by

$$
\begin{aligned}
& L_{7}=2 c_{1} \beta \gamma+2 c_{2}\left(\alpha \gamma-\frac{1}{3} \beta^{2}\right)+2 c_{3} \gamma+c_{4} \alpha+c_{5} \beta+c_{6}, \\
& L_{8}=2 c_{1}\left(2 \gamma^{2}+3 \beta \delta\right)+\frac{2}{3} c_{2}(9 \alpha \delta-\beta \gamma)+6 c_{3} \delta+2 c_{5} \gamma+c_{4} \beta, \\
& L_{9}=18 c_{1} \gamma \delta+2 c_{2}\left(\beta \delta-\frac{1}{3} \gamma^{2}\right)+3 c_{5} \delta+c_{4} \gamma .
\end{aligned}
$$

We consider the following cases. 
4.3.1. $c_{2}=c_{6}=0, c_{1} \neq 0, c_{4} \neq 0$. The general solution of $(70)-(71)$ is

$$
\begin{aligned}
\delta & =\left[D_{1} e^{-c_{4} t}-\frac{18 c_{1}}{c_{4}}\right]^{-1}, \quad \gamma=D_{2} \delta, \\
\alpha & =e^{c_{4} t}\left[\int_{0}^{t} \frac{2 D_{2} C_{4}}{D_{1} c_{4}-18 c_{1} e^{c_{4} t}}\left(c_{1} \beta+c_{3}\right) d t+D_{4}\right], \\
\beta & =\frac{D_{2}^{2} e^{2 c_{4} t}}{3 \eta^{6}}+\frac{18 c_{1} e^{c_{4} t}}{c_{4} \eta}\left[R(\eta)+D_{3}\right],
\end{aligned}
$$

where $\eta=\left(D_{1}-\frac{18 c_{4}}{c_{4}} e^{c_{4} l}\right)^{1 / 3}, \bar{D}_{1}=D_{1}^{1 / 3}$ and

$$
R(\eta)= \begin{cases}\frac{1}{3} \bar{D}_{1}^{-2}\left[\ln \frac{\eta-\bar{D}_{1}}{\sqrt{\eta^{2}+\bar{D}_{1} \eta+\bar{D}_{1}^{2}}}-\sqrt{3} \arctan \frac{\sqrt{3}\left(2 \eta+\bar{D}_{1}\right)}{3 \bar{D}_{1}}\right], & \text { if } D_{1} \neq 0 \\ -\frac{1}{2} \eta^{-2}, & \text { if } \bar{D}_{1}=0\end{cases}
$$

4.3.2. $c_{i}=0, i \neq 2, c_{2} \neq 0$. The general solution of (70)-(71) in this case is given by King [16].

4.3.3. $\quad c_{1} \neq 0, c_{2}=c_{4}=0$. The general solution of (70)-(71) in this subcase is given by

$$
\begin{aligned}
& \alpha=-\frac{D_{2}^{3}}{27 c_{1}}\left(t-t_{0}\right)^{-1}+\frac{D_{2} D_{3}}{3}\left(t-t_{0}\right)^{-\frac{1}{3}}+c_{6}\left(t-t_{0}\right)+D_{4}, \\
& \beta=-\frac{D_{2}^{2}}{3 c_{1}}\left(t-t_{0}\right)^{-1}+D_{3}\left(t-t_{0}\right)^{-\frac{1}{3}}-\frac{c_{3}}{c_{1}}, \\
& \delta=-\frac{1}{18 c_{1}\left(t-t_{0}\right)}, \quad \gamma=D_{2} \delta,
\end{aligned}
$$

which blows up at point $t_{0}$.

4.3.4. $\quad c_{3}=c_{6}=0, c_{1}, c_{2}, c_{4} \neq 0$.

$$
\alpha=\frac{D_{1}^{3}}{27} \delta, \quad \beta=\frac{1}{3} D_{1}^{2} \delta, \quad \gamma=D_{1} \delta, \quad \delta=\left[D_{1} e^{-c_{4} t}-\frac{18 c_{1}}{c_{4}}\right]^{-1},
$$

which with (69) give a separable solution of (1) with (22).

4.4. Under $\sigma_{4}=u_{x x x}$. Here $g$ and $f$ take the form (25) and solving $\sigma_{4}=0$ we obtain the following ansatz for the function $u(t, x)$ :

$$
u(t, x)=\alpha(t)+\beta(t) x+\gamma(t) x^{2} .
$$

The substitution of (75) into (1) with (25) yields that $\alpha, \beta$ and $\gamma$ satisfy

$$
\alpha_{t}=L_{10}(\alpha, \beta, \gamma), \quad \beta_{t}=L_{11}(\alpha, \beta, \gamma), \quad \gamma_{t}=L_{12}(\alpha, \beta, \gamma),
$$


with $L_{10}, L_{11}$ and $L_{12}$ given by

$$
\begin{aligned}
L_{10}= & \frac{1}{2}\left(c_{1} \beta^{2}+c_{3} \beta-2 c_{6} \alpha\right)\left(4 \alpha \gamma-\beta^{2}\right)+\left(2 c_{2} \beta \gamma+2 c_{4} \gamma+c_{7} \beta+c_{5}\right) \beta \\
& +2 c_{8} \alpha \gamma+2 c_{9} \gamma+c_{10} \alpha+c_{11}, \\
L_{11}= & \left(2 c_{1} \beta \gamma+c_{3} \gamma-c_{6} \beta\right)\left(4 \alpha \gamma-\beta^{2}\right)+\left[8 c_{2} \gamma^{2}+\left(4 c_{7}+2 c_{8}\right) \gamma+c_{10}\right] \beta \\
& +4 c_{4} \gamma^{2}+2 c_{5} \gamma, \\
L_{12}= & \left(2 c_{1} \gamma-c_{6}\right)\left(4 \alpha \gamma-\beta^{2}\right) \gamma+\left[8 c_{2} \gamma^{2}+\left(4 c_{7}+2 c_{8}\right) \gamma+c_{10}\right] \gamma .
\end{aligned}
$$

We consider two cases.

4.4.1. $\quad c_{9}=c_{11}=0$. The system (76)-(77) admits an exact solution

$$
\alpha=\frac{\beta^{2}}{4 \gamma}, \quad \beta=\left[4 c_{4} \int_{0}^{t} \gamma d t+D_{1}\right] \gamma
$$

with $\gamma$ solving

$$
\gamma_{t}=8 c_{2} \gamma^{3}+\left(4 c_{7}+2 c_{8}\right) \gamma^{2}+c_{10} \gamma
$$

The general solution of (79) is given in Appendix B.

4.4.2. $c_{i}=0, i \neq 1,3,6$. In this case, we have

$$
\begin{aligned}
& \alpha=\frac{D_{1} \gamma}{4\left(2 c_{1} \gamma-c_{6}\right)}+\frac{\gamma}{4}\left(D_{2}+\frac{c_{3}}{c_{6}} \ln \frac{2 c_{1} \gamma-c_{6}}{\gamma}\right)^{2}, \\
& \beta=D_{2} \gamma+\frac{c_{3}}{c_{6}} \gamma \ln \frac{2 c_{1} \gamma-c_{6}}{\gamma}, \quad \gamma=\left[2 D_{1}\left(t_{0}-t\right)\right]^{-\frac{1}{2}},
\end{aligned}
$$

which blows up at $t_{0}$ and $t_{0}-2 c_{1}^{2} /\left(D_{1} c_{6}^{2}\right)$.

\section{Further examples}

In this section, we give a number of additional examples which provide illustrations that our approach can be extended to consider other types of nonlinear PDEs. The exact solutions of some examples have been constructed by special ansatz on solutions. In $[12,13,16]$, the exact solutions are claimed not to be group-invariant solutions. Our discussion shows that those solutions are invariant under GCSs, which provides a group interpretation for some known results in the literature $[12,13,16]$. 


\subsection{Example 1}

$$
u_{t}=-u^{3} u_{x x x x}+a u^{2} u_{x} u_{x x x}+b u^{2} u_{x x}^{2}+c u u_{x}^{2} u_{x x}+d u_{x}^{4} .
$$

It is easily shown that $\sigma=u_{x x x}$ is a GCS of (81) under $b+2 c+4 d=0$. The corresponding exact solution is

$$
u(t, x)=\alpha(t)+\beta(t) x+\gamma(t) x^{2}
$$

where $\alpha, \beta$ and $\gamma$ satisfy

$$
\begin{aligned}
& \alpha_{t}=2 c \gamma\left(\beta^{2}-4 \alpha \gamma\right) \alpha-d\left(16 \gamma^{2} \alpha^{2}-\beta^{4}\right), \\
& \beta_{t}=2 c \gamma\left(\beta^{2}-4 \gamma \alpha\right) \beta+8 d \beta \gamma\left(\beta^{2}-4 \gamma \alpha\right), \\
& \gamma_{t}=2 c \gamma^{2}\left(\beta^{2}-4 \gamma \alpha\right)+8 d \gamma^{2}\left(\beta^{2}-4 \gamma \alpha\right) .
\end{aligned}
$$

The general solution is as follows.

$$
\text { 5.1.1. } c \neq-10 d / 3 \text { : }
$$

$$
\alpha=\frac{D_{1}^{2}}{4} \gamma-\frac{D_{2}}{4} \gamma^{\frac{c+2 d d}{c+4 d}}, \quad \beta=D_{1} \gamma, \quad \gamma=\left[-2 D_{2}(3 c+10 d)\left(t-t_{0}\right)\right]^{-\frac{c+t d}{3 c+10 d}} .
$$

It blows up at $t_{0}$ provided $(c+4 d)(3 c+10 d)>0$.

$$
\text { 5.1.2. } c=-10 d / 3 \text {. }
$$

$$
\alpha=\frac{D_{1}^{2}}{4} \gamma-\frac{D_{2}}{4} \gamma^{-2}, \quad \beta=D_{1} \gamma, \quad \gamma=\exp \left[\frac{4 d D_{2}}{3}\left(t-t_{0}\right)\right] .
$$

When $a=-\frac{4(2 m+1)}{m-1}, b=-\frac{3(2 m+1)}{m-1}, c=-\frac{6(m+2)(2 m+1)}{(m-1)^{2}}$ and $d=\frac{3(2 m+1)(m+2)}{(m-1)^{3}},(81) \mathrm{can}$ be transformed to

$$
v_{t}=-\frac{1}{m} \frac{\partial^{4}}{\partial x^{4}}\left(v^{4}\right)
$$

by a transformation of dependent variable

$$
v=u^{3 /(m-1)} .
$$

Equation (86) has an exact solution of form (82) if and only if $m=-\frac{1}{2}, m=-\frac{1}{5}$, or $m=-1[16]$. 


\subsection{Example 2}

$$
u_{t}=-\frac{1}{2} \frac{\partial^{4}}{\partial x^{4}}\left(u^{2}\right)
$$

This equation has been discussed in [16]. It is worthy of note that (88) admits two GCSs, $\sigma_{+}=\frac{\partial^{5} u}{\partial x^{5}}$ and $\sigma_{-}=u_{x x x}-\frac{3}{x} u_{x}+\frac{15}{4 x^{2}} u_{x}-\frac{3}{x^{3}} u$. Integrating $\sigma_{+}=0$, we obtain the exact solution of $(88)$ as

$$
u(t, x)=\alpha(t)+\beta(t) x+\gamma(t) x^{2}+\delta(t) x^{3}+\xi(t) x^{4},
$$

where $\alpha, \beta, \gamma, \delta$ and $\xi$ satisfy

$$
\begin{aligned}
& \alpha_{t}=-12\left(\gamma^{2}+2 \beta \delta+2 \alpha \xi\right), \quad \beta_{t}=-120(\beta \xi+\gamma \delta), \\
& \gamma_{t}=-180\left(\delta^{2}+2 \gamma \xi\right), \quad \delta_{t}=-840 \delta \xi, \quad \xi_{t}=-840 \xi^{2},
\end{aligned}
$$

with general solution

$$
\begin{aligned}
& \alpha=\frac{D_{1}^{4}}{215040}\left(t-t_{0}\right)^{-1}+\frac{D_{2} D_{1}^{2}}{16}\left(t-t_{0}\right)^{-\frac{3}{7}}+\frac{D_{1} D_{3}}{4}\left(t-t_{0}\right)^{-\frac{1}{7}}+D_{4}\left(t-t_{0}\right)^{-\frac{2}{70}}, \\
& \beta=\frac{D_{1}^{3}}{13440}\left(t-t_{0}\right)^{-1}+\frac{D_{1} D_{2}}{2}\left(t-t_{0}\right)^{-\frac{3}{7}}+D_{3}\left(t-t_{0}\right)^{-\frac{1}{7}}, \\
& \gamma=\frac{D_{1}^{2}}{2240}\left(t-t_{0}\right)^{-1}+D_{2}\left(t-t_{0}\right)^{-\frac{3}{7}}, \quad \xi=\frac{1}{840}\left(t-t_{0}\right)^{-1}, \quad \delta=D_{1} \xi .
\end{aligned}
$$

This blows up at $t_{0}$. Integrating $\sigma_{-}=0$ gives rise to a formal exact solution of (88)

$$
u(t, x)=\alpha(t) x^{1 / 2}+\beta(t) x^{3 / 2}+\gamma(t) x^{4} .
$$

Substitution of (92) into (88) yields $\alpha, \beta$ and $\gamma$ satisfying

$$
\alpha_{t}=-\frac{945}{8} \alpha \gamma, \quad \beta_{t}=-\frac{3465}{8} \beta \gamma, \quad \gamma_{t}=-1680 \gamma^{2},
$$

with general solution

$$
\alpha=D_{2}\left(t-t_{0}\right)^{-\frac{63}{896}}, \quad \beta=D_{1}\left(t-t_{0}\right)^{-\frac{231}{86}}, \quad \gamma=\frac{1}{1680}\left(t-t_{0}\right)^{-1} .
$$

This also blows up at the finite point $t_{0}$.

\subsection{Example 3}

$$
v_{t}=v \frac{\partial^{2} v}{\partial r^{2}}+\lambda\left(\frac{\partial v}{\partial r}\right)^{2}+\frac{n-1}{r} v \frac{\partial v}{\partial r} .
$$

This is the so-called $N$-dimensional radially symmetric nonlinear diffusion equation discussed by King [16]. We reconsider it using the approach of GCS. Four cases arise in terms of $\lambda$ and $N$. 
5.3.1. $\lambda=-(N+2) / 4$. A direct and tedious calculation shows that $\sigma=$ $v_{r r r}-3 r^{-1} v_{r r}+3 r^{-2} v_{r}$ is a GCS of (95). Integrating $\sigma=0$, we find

$$
v=\alpha(t)+\beta(t) r^{2}+\delta(t) r^{4},
$$

which is compatible with (95) and implies

$$
\alpha_{t}=2 N \alpha \beta, \quad \beta_{t}=(N-2) \beta^{2}+4(N+2) \alpha \delta, \quad \delta_{t}=2 N \beta \delta .
$$

The general solution is given in [16]. It is interesting to note that $\sigma=0$ is the same as the $\Omega$ equation (47) in [22, p. 129].

5.3.2. $\quad N=2, \lambda=-1$. In this case, $\sigma=v_{r r r}-\frac{3}{r} v_{r r}-\frac{p^{2}-7}{r^{2}} v_{r}+\frac{2\left(p^{2}-4\right)}{r^{3}} v$ is a GCS of (95), where $p$ is a constant. Solving $\sigma=0$ we obtain

$$
v(t, r)=\alpha(t) r^{2-p}+\beta(t) r^{2}+\delta(t) r^{p+2} .
$$

The substitution of (98) into (95) with $N=2, \lambda=-1$ gives that $\alpha, \beta$ and $\delta$ satisfy

$$
\alpha_{t}=p^{2} \alpha \beta, \quad \beta_{t}=4 p^{2} \alpha \delta, \quad \delta_{t}=p^{2} \beta \delta .
$$

The solution is also given in [16]. Here $\sigma=0$ corresponds to the $\Omega$ equation (41) of [22, p. 129].

5.3.3. $\lambda=(4-N) /(2(N-3)), N \neq 3$. In this case (95) admits a GCS

$$
\sigma=v_{r r r}+\frac{3(N-3)}{r} v_{r r}+\frac{(2 N-11)(N-3)}{r^{2}} v_{r}-\frac{4(N-4)(N-3)}{r^{3}} v .
$$

Integrating $\sigma=0$ gives

$$
v=\alpha(t) r^{2}+\beta(t) r^{4-N}+\delta(t) r^{6-2 N},
$$

where $\alpha, \beta$ and $\delta$ satisfy

$$
\alpha_{t}=2 \kappa \alpha^{2}, \quad \beta_{t}=2 \kappa \alpha \beta, \quad \delta_{t}=-\frac{1}{2} \kappa\left[(N-4) \beta^{2}+4(3-N) \alpha \delta\right],
$$

which has been derived by Nucci [22] (but note the misprint in [22]). Its general solution is

$$
\begin{aligned}
& \alpha=-\frac{1}{2 \kappa}\left(t-t_{0}\right)^{-1}, \quad \beta=-\frac{D_{1}}{2 \kappa}\left(t-t_{0}\right)^{-1}, \\
& \delta=-\frac{D_{1}^{2}}{8 \kappa}\left(t-t_{0}\right)^{-1}+D_{2}\left(t-t_{0}\right)^{3-N},
\end{aligned}
$$

where $\kappa=(N-2)^{2} /(N-3)$ and $\sigma=0$ is exactly the $\Omega$ equation (51) of [22, p. 129]. This type of solution is new and can be thought of as a generalization of the instantaneous source solution [16]. 
5.3.4. $\lambda=-3 / 4, N=1$. A tedious calculation shows that (95) in this subcase admits a GCS $\sigma=\partial^{5} v / \partial r^{5}$. Integrating $\sigma=0$ we have

$$
v(t, r)=\alpha(t)+\beta(t) r+\gamma(t) r^{2}+\delta(t) r^{3}+\xi(t) r^{4},
$$

the substitution of which into (95) yields the time-dependent function in (69) satisfying

$$
\begin{gathered}
\alpha_{t}=2 \alpha \gamma-\frac{3}{4} \beta^{2}, \quad \beta_{t}=6 \alpha \delta-\beta \gamma, \quad \gamma_{t}=12 \alpha \xi+\frac{3}{2} \beta \delta-\gamma^{2}, \\
\delta_{t}=6 \beta \xi-\gamma \delta, \quad \xi_{t}=2 \gamma \xi-\frac{3}{4} \delta^{2}
\end{gathered}
$$

which has been given in [16] (but note the misprint in [16]). Differentiating (104) once with respect to $t$ leads to

$$
\frac{\alpha_{n}}{\alpha}=\frac{\beta_{n t}}{\beta}=\frac{\gamma_{n t}}{\gamma}=\frac{\delta_{t t}}{\delta}=\frac{\xi_{t t}}{\xi}=2\left(12 \alpha \xi-3 \beta \delta+\gamma^{2}\right)
$$

which combined with (104) gives

$$
\begin{aligned}
& \alpha=\left(D_{1} w+D_{2}\right) \xi, \quad \beta=\left(D_{3} w+D_{4}\right) \xi, \\
& \gamma=\left(D_{5} w+D_{6}\right) \xi, \quad \delta=\left(D_{7} w+D_{8}\right) \xi, \\
& \xi=\left[\frac{3}{4} D_{7}^{2} w^{3}-\frac{3}{2}\left(2 D_{5}-\frac{3}{2} D_{7} D_{8}\right) w^{2}-3\left(2 D_{6}-\frac{3}{4} D_{8}^{2}\right) w+D_{9}\right]^{-1 / 3},
\end{aligned}
$$

where $w$ is determined implicitly by

$$
\int_{w_{0}}^{w}\left[\frac{3}{4} D_{7}^{2} w^{3}-\frac{3}{2}\left(2 D_{5}-\frac{3}{2} D_{7} D_{8}\right) w^{2}-3\left(2 D_{6}-\frac{3}{4} D_{8}^{2}\right) w+D_{9}\right]^{-\frac{2}{3}} d w=t-t_{0} .
$$

Here $D_{i}, i=1,2, \ldots, 9$, satisfy

$$
\begin{aligned}
D_{1} D_{7} D_{8}+D_{3}^{2}-D_{2} D_{7}^{2}-4 D_{1} D_{5} & =0, \\
D_{1} D_{8}^{2}-D_{2} D_{7} D_{8}-4 D_{1} D_{6}+D_{3} D_{4} & =0, \\
D_{4}^{2}-D_{2} D_{8}^{2}+\frac{4}{3} D_{1} D_{9} & =0, \\
D_{7}\left(D_{3} D_{8}-D_{4} D_{7}-8 D_{1}\right) & =0, \\
D_{3} D_{8}^{2}-2 D_{3} D_{6}+2 D_{4} D_{5}-D_{4} D_{7} D_{8}-4 D_{1} D_{8}-4 D_{2} D_{7} & =0, \\
D_{5} D_{8}-D_{6} D_{7}-2 D_{3} & =0, \\
3 D_{6} D_{8}+D_{7} D_{9}-\frac{3}{4} D_{8}^{3}-6 D_{4} & =0,
\end{aligned}
$$




$$
\begin{array}{r}
3 D_{6}^{2}-\frac{3}{4} D_{6} D_{8}^{2}+D_{5} D_{9}-12 D_{2}-\frac{3}{2} D_{4} D_{8}=0 \\
3 D_{4} D_{6}-\frac{3}{4} D_{4} D_{8}^{2}+D_{3} D_{9}-6 D_{2} D_{8}=0 \\
D_{5} D_{8}^{2}-D_{6} D_{7} D_{8}-8 D_{1}-D_{3} D_{8}-D_{4} D_{7}=0
\end{array}
$$

which admits general solution

$$
\begin{aligned}
& D_{1}=\frac{1}{64}\left(4 D_{5} D_{8}^{2}-8 D_{6} D_{7} D_{8}-\frac{3}{4} D_{7}^{2} D_{9}+D_{7} D_{8}^{3}\right), \\
& D_{2}=\frac{1}{64}\left(16 D_{6}^{2}-8 D_{6} D_{8}^{2}+\frac{16}{3} D_{5} D_{9}-\frac{3}{4} D_{7} D_{8} D_{9}+D_{8}^{4}\right), \\
& D_{3}=\frac{1}{2}\left(D_{5} D_{8}-D_{6} D_{7}\right), \quad D_{4}=\frac{1}{24}\left(12 D_{6} D_{8}+4 D_{7} D_{9}-3 D_{8}^{3}\right),
\end{aligned}
$$

where $D_{5}, D_{6}, D_{7}, D_{8}$ and $D_{9}$ are arbitrary constants. Hence (109) is the general solution of (108). We consider several special cases.

(i) $D_{i}=0, i \neq 2,6 . D_{2}=1, D_{6}=2$. The system (104) admits an exact solution

$$
\alpha=\xi, \quad \beta=\delta=0, \quad \gamma=2 \xi, \quad \xi=-\frac{1}{4}\left(t-t_{0}\right)^{-1}
$$

Hence we have

$$
u(t, r)=-\frac{\left(1+r^{2}\right)^{2}}{4\left(t-t_{0}\right)}
$$

which gives a separable solution of (95).

(ii) $D_{1}=D_{3}=D_{5}=D_{7}=D_{9}=0, D_{2}=D_{4}=D_{8}=1, D_{6}=9 / 4$.

The solution of (104) in this case is

$$
\alpha=\beta=\delta=\xi, \quad \gamma=\frac{9}{4} \xi
$$

giving

$$
u=-\frac{4}{15}\left(t-t_{0}\right)^{-1}\left(1+r+\frac{9}{4} r^{2}+r^{3}+r^{4}\right),
$$

which again provides a separable solution for (95).

(iii) $D_{1}=D_{2}=D_{3}=D_{4}=D_{7}=D_{8}=0, D_{5}=D_{6}=1, D_{9}=-3$.

The solution of (104) in this case is

$$
\alpha=\beta=\delta=0, \quad \gamma=-\left(t-t_{0}\right)^{-1}, \quad \xi=\frac{1}{3}\left(t-t_{0}\right)^{2},
$$


so

$$
u=-\left(t-t_{0}\right)^{-1} r^{2}+\frac{1}{3}\left(t-t_{0}\right)^{2} r^{4}
$$

which is exactly the instantaneous source solution [16]. All the above cases are valid for $D_{7}=0$. If $D_{7} \neq 0$, we present the following examples.

(iv) $D_{1}=D_{7}=\frac{2 \sqrt{3}}{3}, D_{2}=1, D_{3}=D_{5}=2 \sqrt{3}, D_{4}=D_{8}=4, D_{6}=6, D_{9}=0$. In this case (104) admits the solution

$$
\begin{array}{ll}
\alpha=\frac{2 \sqrt{3}}{3}-\left(t-t_{0}\right), & \beta=2 \sqrt{3}-4\left(t-t_{0}\right), \\
\gamma=2 \sqrt{3}-6\left(t-t_{0}\right), & \delta=\frac{2 \sqrt{3}}{3}-4\left(t-t_{0}\right), \quad \xi=t_{0}-t,
\end{array}
$$

which gives an unbounded solution to (95).

(v) $D_{1}=D_{5}=D_{9}=0, D_{3}=D_{7}, D_{4}=D_{8}, D_{6}=-2$.

The system (104) possesses the implicit exact solution

$$
\alpha=\xi, \quad \beta=\delta, \quad \gamma=-2 \xi, \quad \xi=\frac{\sqrt{D_{1} \delta-4 \delta^{4}}}{8 \delta}, \quad \int_{\delta_{0}}^{\delta} \frac{d \delta}{\sqrt{D_{1} \delta-4 \delta^{4}}}=t-t_{0}
$$

\subsection{Example 4}

$$
u_{t}=u_{x x}+v_{x}^{2}+v^{2}+a_{1} u+b_{1} v, v_{t}=v_{x x}-u_{x}^{2}-u^{2}+a_{2} u+b_{2} v
$$

It is easy to show that (118) admits the GCS

$$
\sigma_{1}=u_{x x x}+u_{x}, \quad \sigma_{2}=v_{x x x}+v_{x} .
$$

Integrating $\sigma_{1}=\sigma_{2}=0$, we obtain the exact solution of (118) as

$$
\begin{aligned}
& u(t, x)=\alpha_{1}(t)+\beta_{1}(t) \cos x+\gamma_{1}(t) \sin x, \\
& v(t, x)=\alpha_{2}(t)+\beta_{2}(t) \cos x+\gamma_{2}(t) \sin x,
\end{aligned}
$$

which is compatible with (118) and implies $\alpha_{i}, \beta_{i}$ and $\gamma_{i}, i=1,2,3$, satisfy

$$
\begin{aligned}
& \alpha_{1 t}=\alpha_{2}^{2}+\beta_{2}^{2}+\gamma_{2}^{2}+a_{1} \alpha_{1}+b_{1} \alpha_{2}, \beta_{1 t}=2 \alpha_{2} \beta_{2}+\left(a_{1}-1\right) \beta_{1}+b_{1} \beta_{2}, \\
& \gamma_{1 s}=2 \alpha_{2} \gamma_{2}+\left(a_{1}-1\right) \gamma_{1}+b_{1} \gamma_{2}, \quad \alpha_{2 t}=-\alpha_{1}^{2}-\beta_{1}^{2}-\gamma_{1}^{2}+a_{2} \alpha_{1}+b_{2} \alpha_{2}, \\
& \beta_{2 t}=-2 \alpha_{1} \beta_{1}+a_{2} \beta_{1}+\left(b_{2}-1\right) \beta_{2}, \gamma_{2 t}=-2 \alpha_{1} \gamma_{1}+a_{2} \gamma_{1}+\left(b_{2}-1\right) \gamma_{2} .
\end{aligned}
$$

The system (118) was considered by Galaktionov [13] and the particular solution of (120) with $\gamma_{1}=\gamma_{2}=0$ derived by the "method of separation of variables" due to Galaktionov. 


\subsection{Example 5}

$$
\begin{aligned}
& u_{t}=u_{x x}+v_{x} u_{x}+u v+a_{1} u+b_{1} v, \\
& v_{t}=v v_{x x}+a_{2} v_{x}^{2}+b_{2} v^{2}+a_{3} u+b_{3} v .
\end{aligned}
$$

Here $a_{i}, b_{i}, i=1,2$, are constants. This is a parabolic system of quasilinear equations of nonlinear heat conduction type with source, which is more general than Galaktionov's example 6 of p. 869 in [13]. We find that (122) also admits the GCS (119) under $b_{2}=a_{2}+1$, so that the exact solution of (122) is given by (120), instead of the system (121). Here $\alpha_{i}, \beta_{i}$ and $\gamma_{i}$ satisfy

$$
\begin{aligned}
& \alpha_{1 t}=\alpha_{1} \alpha_{2}+\beta_{1} \beta_{2}+\gamma_{1} \gamma_{2}+a_{1} \alpha_{1}+b_{1} \alpha_{2}, \\
& \alpha_{2 t}=b_{2} \alpha_{2}^{2}+a_{2} \beta_{2}^{2}+a_{2} \gamma_{2}^{2}+b_{3} \alpha_{2}+a_{3} \alpha_{1}, \\
& \beta_{1 t}=\alpha_{2} \beta_{1}+\alpha_{1} \beta_{2}+\left(a_{1}-1\right) \beta_{1}+b_{1} \beta_{2}, \\
& \beta_{2 t}=\left(2 a_{2}+1\right) \alpha_{2} \beta_{2}+b_{3} \beta_{2}+a_{3} \beta_{1}, \\
& \gamma_{1 t}=\alpha_{1} \gamma_{2}+\alpha_{2} \gamma_{1}+\left(a_{1}-1\right) \gamma_{1}+b_{1} \gamma_{2}, \\
& \gamma_{2 t}=\left(2 a_{2}+1\right) \alpha_{2} \gamma_{2}+b_{3} \gamma_{2}+a_{3} \gamma_{1} .
\end{aligned}
$$

\subsection{Example 6}

$$
u_{t t}=u_{x x x x}+u_{x x}+\left(u^{2}\right)_{x x}-u^{2}+a u, a=\text { const. }
$$

It is easily shown that (124) admits a GCS $\sigma=u_{x x x}-\frac{u_{x}}{4}$. Solving $\sigma=0$ we have an exact solution of (124) as

$$
u(t, x)=\alpha(t)+\beta(t) \cosh \left(\frac{x}{2}\right)+\gamma(t) \sinh \left(\frac{x}{2}\right),
$$

where $\alpha, \beta$ and $\gamma$ satisfy

$$
\begin{gathered}
\alpha_{t t}=-\alpha^{2}-\beta^{2}-\gamma^{2}+a \alpha, \quad \beta_{t t}=-\frac{3}{2} \alpha \beta+\left(a+\frac{5}{16}\right) \beta \\
\gamma_{t t}=-\frac{3}{2} \alpha \gamma+\left(a+\frac{5}{16}\right) \gamma .
\end{gathered}
$$

\subsection{Example 7}

$$
u_{t}=u_{x x x x}+u_{x x}+u_{x}^{2}+u^{2}+a u, a=\text { const. }
$$

Equation (127) admits a GCS $\sigma=u_{x x x}+u_{x}$. Integrating $\sigma=0$ we obtain

$$
u(t, x)=\alpha(t)+\beta(t) \cos x+\gamma(t) \sin x,
$$

where $\alpha, \beta$ and $\gamma$ satisfy

$$
\alpha_{t}=\alpha^{2}+\beta^{2}+\gamma^{2}+a \alpha, \quad \beta_{t}=2 \alpha \beta+a \beta, \quad \gamma_{t}=2 \alpha \gamma+a \gamma .
$$




\subsection{Example 8}

$$
u_{t}=(-1)^{m+1}\left(\frac{\partial^{2}}{\partial x^{2}}\right)^{m} u+u_{x}^{2}+u^{2}+a u, a=\text { const. }
$$

This is a semilinear parabolic equation of $2 \mathrm{~m}$-th order. It is easily shown that $\sigma=u_{x x x}+u_{x}$ also is a GCS of (130), so its exact solution takes the form (128), in which $\alpha, \beta$ and $\gamma$ satisfy

$$
\alpha_{t}=\alpha^{2}+\beta^{2}+\gamma^{2}+a \alpha, \quad \beta_{t}=2 \alpha \beta+(a-1) \beta, \quad \gamma_{t}=2 \alpha \gamma+(a-1) \gamma .
$$

Equation (130) is discussed in [13]. It is interesting to notice that the exact solution of (130) is independent of $m$.

\subsection{Example 9}

$$
u_{t}=u_{x x}+v_{x} u_{x}-u v+a_{1} u+b_{1} v, \quad v_{t}=v v_{x x}+a_{2} v_{x}^{2}+b_{2} v^{2}+a_{3} u+b_{3} v .
$$

Analogously to (122), one can show that (132) admits the GCS $\sigma_{1}=u_{x x x}-u_{x}$, $\sigma_{2}=v_{x x x}-v_{x}$ under $b_{2}=a_{2}+1$. Solving $\sigma_{1}=\sigma_{2}=0$ gives rise to the exact solution of (132) as

$$
\begin{aligned}
& u(t, x)=\alpha_{1}(t)+\beta_{1} \cosh x+\gamma_{1} \sinh x, \\
& v(t, x)=\alpha_{2}(t)+\beta_{2} \cosh x+\gamma_{2} \sinh x .
\end{aligned}
$$

The substitution of (133) into (132) yields that $\alpha_{i}, \beta_{i}$ and $\gamma_{i}, i=1,2$, satisfy

$$
\begin{aligned}
& \alpha_{1 t}=-\alpha_{1} \alpha_{2}-\beta_{1} \beta_{2}-\gamma_{1} \gamma_{2}+a_{1} \alpha_{1}+b_{1} \alpha_{2}, \\
& \beta_{1 t}=-\alpha_{1} \beta_{2}-\alpha_{2} \beta_{1}+\left(a_{1}+1\right) \beta_{1}+b_{1} \beta_{2}, \\
& \gamma_{1 t}=-\alpha_{1} \gamma_{2}-\alpha_{2} \gamma_{1}+\left(a_{1}+1\right) \gamma_{1}+b_{1} \gamma_{2}, \\
& \alpha_{2 t}=-b_{2} \alpha_{2}^{2}-a_{2} \beta_{2}^{2}+a_{2} \gamma_{2}^{2}+a_{3} \alpha_{1}+b_{3} \alpha_{2}, \\
& \beta_{2 t}=\left(-2 a_{2}-1\right) \alpha_{2} \beta_{2}+a_{3} \beta_{1}+b_{3} \beta_{2}, \\
& \gamma_{2 t}=-\left(2 a_{2}+1\right) \alpha_{2} \gamma_{2}+a_{3} \gamma_{1}+b_{3} \gamma_{2} .
\end{aligned}
$$

\subsection{Example 10}

$$
u_{t}=u_{x x}+v_{x}^{2}-v^{2}+a_{1} u+b_{1} v, \quad v_{t}=v_{x x}-u_{x}^{2}+u^{2}+a_{2} u+b_{2} v .
$$

Similarly to (118), one can show that this admits the GCS $\sigma_{1}=u_{x x x}-u_{x}, \sigma_{2}=$ $v_{x x x}-v_{x}$. Integrating $\sigma_{1}=\sigma_{2}=0$, we obtain the exact solution of (135) as

$$
u(t, x)=\alpha_{1}(t)+\beta_{1}(t) \cosh x+\gamma_{1}(t) \sinh x,
$$


TABLE 1.

\begin{tabular}{|c|c|c|c|c|}
\hline$c_{i}, i=9,10$ & $F_{1}$ & $F_{2}$ & $F_{3}$ & $F_{4}$ \\
\hline$c_{10}>c_{9}, c_{9}>0$ & US & AS & US & US \\
\hline$c_{10}<-c_{9}, c_{9}>0$ & AS & US & US & US \\
\hline$c_{10}>-c_{9}, c_{9} \leq 0$ & US & AS & US & US \\
\hline$c_{10}<c_{9}, c_{9} \leq 0$ & AS & US & US & US \\
\hline
\end{tabular}

TABLE 2.

\begin{tabular}{|c|c|c|}
\hline$c_{i}, i=9,10$ & $F_{1}$ & $F_{2}$ \\
\hline$c_{10}<0, c_{9}>0$ & AS & US \\
\hline$c_{10}>0, c_{9}>0$ & US & AS \\
\hline$c_{9}<0$ & US & US \\
\hline
\end{tabular}

$$
v(t, x)=\alpha_{2}(t)+\beta_{2}(t) \cosh x+\gamma_{2}(t) \sinh x,
$$

which combined with (135) implies $\alpha_{i}, \beta_{i}$ and $\gamma_{i}, i=1,2$, satisfy

$$
\begin{array}{ll}
\alpha_{1 t}=-\alpha_{2}^{2}-\beta_{2}^{2}+\gamma_{2}^{2}+a_{1} \alpha_{1}+b_{1} \alpha_{2}, & \beta_{1 t}=-2 \alpha_{2} \beta_{2}+\left(a_{1}+1\right) \beta_{1}+b_{1} \beta_{2}, \\
\gamma_{1 t}=-2 \alpha_{2} \gamma_{2}+\left(a_{1}+1\right) \gamma_{1}+b_{1} \gamma_{2}, & \alpha_{2 t}=\alpha_{1}^{2}+\beta_{1}^{2}-\gamma_{1}^{2}+a_{2} \alpha_{1}+b_{2} \alpha_{2}, \\
\beta_{2 t}=2 \alpha_{1} \beta_{1}+a_{2} \beta_{1}+\left(b_{2}+1\right) \beta_{2}, & \gamma_{2 t}=2 \alpha_{1} \gamma_{1}+a_{2} \gamma_{1}+\left(b_{2}+1\right) \gamma_{2} .
\end{array}
$$

\section{Dynamical behavior of a system of ODEs}

As mentioned in Section 5, it is very difficult to obtain exact solutions of some ODEs in Section 4. So an important problem is to study the dynamical behavior of these ODEs. Since (in two dimensions) chaos is possible in the nonautonomous but not the autonomous case, we focus primarily on the stability of fixed points and qualitative properties of the system.

Let us consider a general autonomous vector field

$$
y_{t}=Y(y), \quad y \in R^{n} .
$$

A fixed point of (138) is a point $\bar{y} \in R^{n}$ such that

$$
Y(\bar{y})=0,
$$

that is, a solution does not change in time.

Let $\bar{y}(t)$ be any solution of (138). Roughly speaking, $\bar{y}(t)$ is stable solution if all nearby solutions stay nearby for all future time. It is asymptotically stable if nearby solutions actually converge to $\bar{y}(t)$ as $t \rightarrow \infty$. A mathematical definition is provided in $[14,28]$. 
DEFINITION 6.1. (Liapunov stability.) $\bar{y}(t)$ is said to be stable if, given $\epsilon>0$, there exists a $\delta=\delta(\epsilon)>0$ such that, for any other solution $y(t)$ of (138) satisfying

$$
\left|\bar{y}\left(t_{0}\right)-y\left(t_{0}\right)\right|<\delta, \text { then }|\bar{y}(t)-y(t)|<\epsilon,
$$

for $t>t_{0}, t_{0} \in R$. A solution that is not stable is said to be unstable.

DEFINITION 6.2. (Asymptotic stability.) $\bar{y}(t)$ is said to be asymptotically stable if it is Liapunov stable and if there exists a constant $b>0$ such that if $\left|\bar{y}\left(t_{0}\right)-y\left(t_{0}\right)\right|<b$, then $\lim _{t \rightarrow \infty}|\bar{y}(t)-y(t)|=0$.

It is easy to see that if a solution $\bar{y}$ is asymptotically stable, then is stable. Denoting the derivative of $f$ by $D f(x)$, we have the following [14].

PROPOSITION 6.1. Suppose all the eigenvalues of DY $(\bar{y})$ have negative real parts. Then the fixed points $y=\bar{y}$ of the nonlinear vector field are asymptotically stable.

To study the qualitative properties of the system of ODEs, we focus attention on

$$
\begin{aligned}
& \alpha_{t}=X_{m}(\alpha, \beta)+\Phi(\alpha, \beta), \\
& \beta_{t}=Y_{n}(\alpha, \beta)+\Psi(\alpha, \beta),
\end{aligned}
$$

where $X_{m}$ and $Y_{n}$ are respectively homogeneous polynomials of $\alpha$ and $\beta$ of orders $m$ and $n$. The lowest order of $\Phi$ and $\Psi$ are respectively $m+1$ and $n+1$. Assume the origin $(0,0)$ is an isolated fixed point. Introducing the polar coordinates $\alpha=$ $r \cos \theta, \beta=r \sin \theta$, a straightforward calculation shows that

$$
\frac{1}{r} \frac{d r}{d \theta}=\frac{H(\theta)+O(r)}{G(\theta)+O(r)}
$$

The following definition is useful.

Definition 6.3. The equation $G(\theta)=0$ is called the characteristic equation of (140). The direction $\theta$ determined by the characteristic equation is called the special direction of (140).

To illustrate our approach, we consider three special cases of (27)-(28).

6.1. $\quad c_{i}=0, i \neq 7,9,10, c_{7}=1$. The system (27)-(28) then reads

$$
\begin{aligned}
\alpha_{t} & =\alpha^{2}+\beta^{2}+\gamma^{2}+c_{10} \alpha, \\
\beta_{t} & =2 \alpha \beta+\left(c_{10}-c_{9}\right) \beta, \\
\gamma_{t} & =2 \alpha \gamma+\left(c_{10}-c_{9}\right) \gamma .
\end{aligned}
$$


It is easy to see that there exists an arbitrary constant $d$ such that $\gamma=d \beta$. Hence (141) is equivalent to

$$
\begin{aligned}
\alpha_{t} & =\alpha^{2}+\left(1+d^{2}\right) \beta^{2}+c_{10} \alpha, \\
\beta_{t} & =2 \alpha \beta+\left(c_{10}-c_{9}\right) \beta,
\end{aligned}
$$

which has been derived by Galaktionov [13]. Six subcases arise.

6.1.1. $\quad c_{10}^{2}>c_{9}^{2}, c_{10} \neq 0$. In this subcase, (142) has four fixed points denoted by $F_{i}(\alpha, \beta), i=1,2,3,4$, with eigenvalues $\lambda_{1,2}$ of the linearization about each fixed point given by

$$
\begin{aligned}
& F_{1}(0,0) \quad \Rightarrow \quad \lambda_{1}=c_{10}, \lambda_{2}=c_{10}-c_{9}, \\
& F_{2}\left(-c_{10}, 0\right) \quad \Rightarrow \quad \lambda_{1}=-c_{10}, \lambda_{2}=-c_{10}-c_{9} \text {, } \\
& F_{3}\left(\frac{c_{9}-c_{10}}{2}, \frac{\sqrt{c_{10}^{2}-c_{9}^{2}}}{2 \sqrt{1+d^{2}}}\right) \Rightarrow \lambda_{1,2}=\frac{c_{9} \pm \sqrt{4 c_{10}^{2}-3 c_{9}^{2}}}{2}, \\
& F_{4}\left(\frac{c_{9}-c_{10}}{2},-\frac{\sqrt{c_{10}^{2}-c_{9}^{2}}}{2 \sqrt{1+d^{2}}}\right) \Rightarrow \lambda_{1,2}=\frac{c_{9} \pm \sqrt{4 c_{10}^{2}-3 c_{9}^{2}}}{2} .
\end{aligned}
$$

The stability of $F_{i}, i=1,2,3,4$, is indicated in Table 1 under assumptions on $c_{i}$, $i=9,10$. In the tables, we use "US", "S" and "AS" to denote unstable, stable and asymptotically stable respectively.

6.1.2. $c_{10}^{2}<c_{9}^{2}$. The system (142) has two fixed points $F_{1}(0,0)$ and $F_{2}\left(-c_{10}, 0\right)$. The stability is pointed out in Table 2 .

6.1.3. $c_{10}=c_{9}=0$. In this subcase (142) has exactly one fixed point $F_{1}(0,0)$. Equation (140) in this case then becomes

$$
\frac{1}{r} \frac{d r}{d \theta}=\frac{\cos \theta\left[1+\left(2+d^{2}\right) \sin ^{2} \theta\right]+O(r)}{\sin \theta\left[\cos ^{2} \theta-\left(1+d^{2}\right) \sin ^{2} \theta\right]+O(r)},
$$

which has six special directions $\theta_{1}=0, \theta_{2}=\pi, \theta_{3}=\arctan \left(1 / \sqrt{1+d^{2}}\right), \theta_{4}=\pi+\theta_{3}$, $\theta_{5}=\pi-\theta_{3}$ and $\theta_{6}=-\theta_{3}$. We have the following results [31, Theorems 3.4-3.7].

THEOREM 6.1. The origin $(0,0)$ is exactly one fixed point of the system (142). Along each of the directions $\theta_{1}, \theta_{2}, \theta_{4}$ and $\theta_{5}$, there are infinite orbits reaching to $(0,0)$. Along each of directions $\theta_{3}$ and $\theta_{6}$, there is only one orbit reaching to $(0,0)$.

6.1.4. $\quad c_{10}=c_{9} \neq 0$. There are two fixed points $F_{1}(0,0)$ and $F_{2}\left(-c_{10}, 0\right)$. It is easy to see that $F_{2}$ is asymptotically stable when $c_{10}>0$ and unstable when $C_{10}<0$. For the fixed point $F_{1}(0,0)$, analogous to the above discussion, we have the following.

THEOREM 6.2. There is only one orbit reaching to $(0,0)$ along each of two special directions $\theta_{1}=0$ and $\theta=\pi$. 
6.1.5. $c_{10}=-c_{9} \neq 0$. In this subcase, $(142)$ has two fixed points $F_{1}(0,0)$ and $F_{2}\left(-c_{10}, 0\right)$. For $F_{1}(0,0)$, when $C_{10}>0, F_{1}$ is unstable and when $C_{10}<0, F_{1}$ is asymptotically stable. For $F_{2}\left(-c_{10}, 0\right)$, there are four special directions $\theta_{1}=0$, $\theta_{2}=\pi / 2, \theta_{3}=\pi$ and $\theta_{4}=3 \pi / 2$. Here we have the following.

THEOREM 6.3. Along each of special directions $\theta_{1}$ and $\theta_{2}$, there are infinite orbits which are tangent to $\alpha=-c_{10}$ reaching to point $F_{2}$. Along each of special directions $\theta_{3}$ and $\theta_{4}$, there is exactly one orbit which is tangent to $\alpha=-c_{10}$, reaching to point $F_{2}$.

6.1.6. $\quad c_{10}=0, c_{9} \neq 0$. In this subcase, (142) has only one fixed point $F_{1}(0,0)$. In the neighbourhood of $F_{1}(0,0)$, we have the following theorem.

THEOREM 6.4. Along each of two special directions $\theta_{1}=\pi / 2$ and $\theta_{2}=3 \pi / 2$, there is only one orbit reaching to the origin $(0,0)$.

6.2. $c_{i}=0, i \neq 2,8,10$. As in case 6.1 , it suffices to consider

$$
\begin{aligned}
\alpha_{t} & =\left[2 c_{2} \alpha^{2}+c_{8} \alpha+c_{10}\right] \alpha, \\
\beta_{t} & =\left[3 c_{2} \alpha^{2}-c_{2}\left(1+d^{2}\right) \beta^{2}+c_{8} \alpha+c_{10}\right] \beta,
\end{aligned}
$$

where $d$ is an arbitrary constant. We distinguish five subcases.

6.2.1. $c_{2} c_{10}>0, c_{8}^{2}-8 c_{2} c_{10}>0$. In this subcase, there are nine fixed points with eigenvalues $\lambda_{1,2}$ of the linearization about each fixed point given by

$$
\begin{gathered}
F_{1}(0,0) \Rightarrow \lambda_{1}=\lambda_{2}=c_{10}, \\
F_{2}\left(0, \sqrt{\frac{c_{10}}{\left(1+d^{2}\right) c_{2}}}\right) \Rightarrow \lambda_{1}=c_{10}, \quad \lambda_{2}=-2 c_{10}, \\
F_{3}\left(0,-\sqrt{\frac{c_{10}}{\left(1+d^{2}\right) c_{2}}}\right) \Rightarrow \lambda_{1}=c_{10}, \quad \lambda_{2}=-2 c_{10}, \\
F_{4}\left(\frac{-c_{8}+\sqrt{c_{8}^{2}-8 c_{2} c_{10}}}{4 c_{2}}, 0\right) \\
\Rightarrow \lambda_{1}=\frac{c_{8}^{2}-8 c_{2} c_{10}-c_{8} \sqrt{c_{8}^{2}-8 c_{2} c_{10}}}{4 c_{2}}, \quad \lambda_{2}=\frac{c_{8}^{2}-8 c_{2} c_{10}-c_{8} \sqrt{c_{8}^{2}-8 c_{2} c_{10}}}{8 c_{2}}, \\
F_{5}\left(\frac{-c_{8}-\sqrt{c_{8}^{2}-8 c_{2} c_{10}}}{4 c_{2}}, 0\right) \\
\Rightarrow \lambda_{1}=\frac{c_{8}^{2}-8 c_{2} c_{10}+c_{8} \sqrt{c_{8}^{2}-8 c_{2} c_{10}}}{4 c_{2}}, \quad \lambda_{2}=\frac{c_{8}^{2}-8 c_{2} c_{10}+c_{8} \sqrt{c_{8}^{2}-8 c_{2} c_{10}}}{8 c_{2}}
\end{gathered}
$$




$$
\begin{aligned}
& F_{6}\left(\frac{-c_{8}+\sqrt{c_{8}^{2}-8 c_{2} c_{10}}}{4 c_{2}},-\frac{-c_{8}+\sqrt{c_{8}^{2}-8 c_{2} c_{10}}}{4 c_{2} \sqrt{1+d^{2}}}\right) \\
& \left.\quad \Rightarrow \lambda_{1}=\frac{c_{8}^{2}-8 c_{2} c_{10}-c_{8} \sqrt{c_{8}^{2}-8 c_{2} c_{10}}, \quad \lambda_{2}=-2 c_{2}\left[\frac{-c_{8}+\sqrt{c_{8}^{2}-8 c_{2} c_{10}}}{4 c_{2}}\right]^{2},}{4 c_{2}}, \frac{-c_{8}+\sqrt{c_{8}^{2}-8 c_{2} c_{10}}}{4 c_{2} \sqrt{1+d^{2}}}\right) \\
& F_{7}\left(\frac{-c_{8}+\sqrt{c_{8}^{2}-8 c_{2} c_{10}}}{4 c_{2}}, \quad \lambda_{2}=-2 c_{2}\left[\frac{-c_{8}+\sqrt{c_{8}^{2}-8 c_{2} c_{10}}}{4 c_{2}}\right]^{2},\right. \\
& \left.\quad \Rightarrow \lambda_{1}=\frac{c_{8}^{2}-8 c_{2} c_{10}-c_{8} \sqrt{c_{8}^{2}-8 c_{2} c_{10}}}{4 c_{2}}, \frac{-c_{8}-\sqrt{c_{8}^{2}-8 c_{2} c_{10}}}{4 c_{2} \sqrt{1+d^{2}}}\right) \\
& F_{8}\left(\frac{-c_{8}-\sqrt{c_{8}^{2}-8 c_{2} c_{10}}}{4 c_{2}}, \quad \lambda_{2}=-2 c_{2}\left[\frac{-c_{8}-\sqrt{c_{8}^{2}-8 c_{2} c_{10}}}{4 c_{2}}\right]^{2}\right. \\
& \quad \Rightarrow \lambda_{1}=\frac{c_{8}^{2}-8 c_{2} c_{10}+c_{8} \sqrt{c_{8}^{2}-8 c_{2} c_{10}}}{4 c_{2}}, \\
& F_{9}\left(\frac{-c_{8}-\sqrt{c_{8}^{2}-8 c_{2} c_{10}}}{4 c_{2}}, \frac{c_{8}+\sqrt{c_{8}^{2}-8 c_{2} c_{10}}}{4 c_{2}}\right) \\
& \quad \frac{c_{8}^{2}-8 c_{2} c_{10}+c_{8} \sqrt{c_{8}^{2}-8 c_{2} c_{10}}}{4 c_{2}},
\end{aligned}
$$

6.2.2. $c_{2} c_{10}>0, c_{8}^{2}-8 c_{2} c_{10}<0$. The system (144) has three fixed points $F_{1}, F_{2}$ and $F_{3}$.

6.2.3. $c_{2} c_{10}>0, c_{8}^{2}=8 c_{2} c_{10}$. In this subcase, (144) has six fixed points $F_{4}^{1}\left(-c_{8} /\left(4 c_{2}\right), 0\right), \quad F_{5}^{1}\left(-c_{8} /\left(4 c_{2}\right),-c_{8} /\left(4 c_{2} \sqrt{1+d^{2}}\right)\right)$ and $F_{6}^{1}\left(-c_{8} /\left(4 c_{2}\right), c_{8} /\left(4 c_{2}\right.\right.$ $\left.\left.\sqrt{1+d^{2}}\right)\right)$ in addition to $F_{1}, F_{2}$ and $F_{3}$.

6.2.4. $c_{10}=0, c_{2}, c_{8} \neq 0$. The system (144) has four fixed points, $F_{1}$, $F_{2}^{2}\left(-c_{8} /\left(2 c_{2}\right), 0\right), \quad F_{3}^{2}\left(-c_{8} /\left(2 c_{2}\right), c_{8} /\left(2 c_{2} \sqrt{1+d^{2}}\right)\right)$ and $F_{4}^{2}\left(-c_{8} /\left(2 c_{2}\right),-c_{8} /\left(2 c_{2}\right.\right.$ $\left.\left.\sqrt{1+d^{2}}\right)\right)$.

6.2.5. $c_{2} c_{10}<0$. In this subcase, (144) admits seven fixed points $F_{i}, i=$ $1,4,5,6,7,8,9$. The stability of $F_{i}, i=1,2, \ldots, 9$ is indicated in Table 3 . In this table $\Delta=c_{8}^{2}-8 c_{2} c_{10}$.

For the fixed points $F_{4}^{1}, F_{5}^{1}$ and $F_{6}^{1}$, we have the following common qualitative property.

THEOREM 6.5. Along each of two special directions $\theta_{1}=\pi / 2$ and $\theta_{2}=3 \pi / 2$, there is only one orbit, which is tangent to $\alpha=-c_{8} /\left(4 c_{2}\right)$, reaching to points $F_{4}^{i}$, $i=4,5,6$. 
TABLE 3.

\begin{tabular}{|c|c|c|c|c|c|c|c|c|c|c|c|c|}
\hline$\Delta$ & $c_{10}$ & $c_{2}$ & $c_{8}$ & $F_{1}$ & $F_{2}$ & $F_{3}$ & $F_{4}$ & $\overline{F_{5}}$ & $F_{6}$ & $F_{7}$ & $F_{8}$ & $F_{9}$ \\
\hline \multirow[t]{2}{*}{$\Delta<0$} & $c_{10}<0$ & $c_{2}<0$ & arbitrary & AS & US & US & & & & & & \\
\hline & $c_{10}>0$ & $c_{2}>0$ & arbitrary & US & US & US & & & & & & \\
\hline \multirow{8}{*}{$\Delta>0$} & $c_{10}<0$ & $c_{2}>0$ & $c_{8}<0$ & AS & & & US & AS & US & US & US & US \\
\hline & $c_{10}>0$ & $c_{2}>0$ & $c_{8}<0$ & US & US & US & US & US & US & US & AS & $\overline{\mathrm{AS}}$ \\
\hline & $c_{10}>0$ & $c_{2}>0$ & $c_{8}>0$ & US & US & US & US & AS & $\mathrm{AS}$ & AS & US & US \\
\hline & $c_{10}<0$ & $c_{2}>0$ & $c_{8}>0$ & AS & & & $\mathrm{AS}$ & US & US & US & US & US \\
\hline & $c_{10}<0$ & $c_{2}<0$ & $c_{8}<0$ & AS & US & US & US & US & US & US & US & US \\
\hline & $c_{10}>0$ & $c_{2}<0$ & $c_{8}<0$ & US & & & US & AS & US & $\overline{\text { US }}$ & US & US \\
\hline & $c_{10}<0$ & $c_{2}<0$ & $c_{8}>0$ & AS & US & $\overline{\text { US }}$ & US & US & US & $\overline{\text { US }}$ & US & US \\
\hline & $c_{10}>0$ & $c_{2}<0$ & $c_{8}>0$ & US & & & AS & US & US & US & US & US \\
\hline
\end{tabular}

When $c_{8}^{2}-8 c_{2} c_{10}=0$ and $c_{2} c_{10}>0$, the stability of fixed points $F_{1}, F_{2}^{2}, F_{3}^{2}$ and $F_{4}^{2}$ is indicated in Table 4.

6.3. $c_{i}=0, i \neq 4,6,10$.

In this case, (27)-(28) is equivalent to

$$
\begin{aligned}
\alpha_{t} & =c_{6}\left[\left(1+d^{2}\right) \beta^{2}-\alpha^{2}\right] \alpha+c_{7}\left[\alpha^{2}+\left(1+d^{2}\right) \beta^{2}\right]+c_{10} \alpha, \\
\beta_{t} & =\left[c_{6}\left(\left(1+d^{2}\right) \beta^{2}-\alpha^{2}\right)+2 c_{7} \alpha+c_{10}\right] \beta,
\end{aligned}
$$

where $d$ is an arbitrary constant. To obtain the fixed points, we consider four subcases.

6.3.1. $c_{10} \neq 0, c_{7}^{2}+4 c_{6} c_{10}>0$. In this subcase, (145) has five fixed points denoted by $E_{i}(\alpha, \beta), i=1, \ldots, 5$, with eigenvalues $\lambda_{1,2}$ of the linearization about the fixed points

$$
\begin{aligned}
& E_{1}(0,0) \Rightarrow \lambda_{1}=\lambda_{2}=c_{10} \\
& E_{2}\left(\frac{c_{7}+\sqrt{c_{7}^{2}+4 c_{6} c_{10}}}{2 c_{6}}, 0\right) \Rightarrow \lambda_{1}=-\frac{c_{7}^{2}+4 c_{6} c_{10}+c_{7} \sqrt{c_{7}^{2}+4 c_{6} c_{10}}}{2 c_{6}}, \\
& \lambda_{2}=\frac{c_{7}\left(c_{7}+\sqrt{c_{7}^{2}+4 c_{6} c_{10}}\right)}{2 c_{6}}, \\
& E_{3}\left(\frac{c_{7}-\sqrt{c_{7}^{2}+4 c_{6} c_{10}}}{2 c_{6}}, 0\right) \Rightarrow \lambda_{1}=-\frac{c_{7}^{2}+4 c_{6} c_{10}-c_{7} \sqrt{c_{7}^{2}+4 c_{6} c_{10}}}{2 c_{6}}, \\
& E_{4}\left(-\frac{c_{10}}{2 c_{7}},-\frac{c_{10}}{2 c_{7} \sqrt{1+d^{2}}}\right) \Rightarrow \lambda_{1,2}= \pm c_{10},
\end{aligned}
$$


TABLE 4.

\begin{tabular}{|c|c|c|c|c|c|}
\hline$c_{2}$ & $c_{8}$ & $F_{1}$ & $F_{2}^{2}$ & $F_{3}^{2}$ & $F_{4}^{2}$ \\
\hline$c_{2}>0$ & $c_{8}<0$ & $\mathrm{US}$ & $\mathrm{US}$ & $\mathrm{US}$ & $\mathrm{US}$ \\
\cline { 2 - 6 } & $c_{8}>0$ & $\mathrm{~S}$ & $\mathrm{US}$ & $\mathrm{US}$ & $\mathrm{US}$ \\
\hline \multirow{2}{*}{$c_{2}<0$} & $c_{8}<0$ & $\mathrm{~S}$ & $\mathrm{~S}$ & $\mathrm{~S}$ & $\mathrm{~S}$ \\
\cline { 2 - 6 } & $c_{8}>0$ & $\mathrm{US}$ & $\mathrm{S}$ & $\mathrm{S}$ & $\mathrm{S}$ \\
\hline
\end{tabular}

$$
E_{5}\left(-\frac{c_{10}}{2 c_{7}}, \frac{c_{10}}{2 c_{7} \sqrt{1+d^{2}}}\right) \Rightarrow \lambda_{1,2}= \pm c_{10}
$$

6.3.2. $\quad c_{10} \neq 0, c_{7}^{2}+4 c_{6} c_{10}<0$. The system (145) has three fixed points $E_{1}, E_{4}$ and $E_{5}$.

6.3.3. $c_{10}, c_{7} \neq 0, c_{7}^{2}+4 c_{6} c_{10}=0$. There are four fixed points $E_{1}, E_{4}, E_{5}$ and $E_{3}^{1}\left(c_{7} /\left(2 c_{6}\right), 0\right)$.

6.3.4. $c_{10}=0, c_{6}, c_{7} \neq 0$. In this subcase, (145) has two fixed points $E_{1}$ and $E_{2}^{2}\left(c_{7} / c_{6}, 0\right)$.

When $c_{10} \neq 0$, the stability of the fixed points $E_{i}, i=1,2, \ldots, 5$ is indicated in Table 5. In this table $\Omega=c_{7}^{2}+4 c_{6} c_{10}$.

For the fixed point $E_{3}^{1}$, we have the following.

THEOREM 6.6. Along each of two special directions $\theta_{1}=\pi / 2$ and $\theta_{2}=3 \pi / 2$, there exists only one orbit reaching to point $E_{3}^{1}$.

When $c_{10}=0$ and $c_{6}, c_{7} \neq 0$, the fixed point $E_{2}^{2}$ is unstable. For the fixed point $E_{1}(0,0)$, there are six special directions

$$
\begin{gathered}
\theta_{1}=0, \quad \theta_{2}=\pi, \quad \theta_{3}=\arctan \frac{1}{\sqrt{1+d^{2}}}, \quad \theta_{4}=-\arctan \frac{1}{\sqrt{1+d^{2}}}, \\
\theta_{5}=\pi-\arctan \frac{1}{\sqrt{1+d^{2}}}, \quad \theta_{6}=\pi+\arctan \frac{1}{\sqrt{1+d^{2}}} .
\end{gathered}
$$

We have the following theorem.

THEOREM 6.7. Along each of special directions $\theta_{1}$ and $\theta_{2}$, there are infinite orbits reaching to point $E_{1}$. Along each of special directions $\theta_{i}, i=3,4,5,6$, there is only one orbit reaching to point $E_{1}$. 
TABLE 5.

\begin{tabular}{|c|c|c|c|c|c|c|c|c|}
\hline$\Omega$ & $c_{6}$ & $c_{7}$ & $c_{10}$ & $E_{1}$ & $E_{2}$ & $E_{3}$ & $E_{4}$ & $E_{5}$ \\
\hline$\Omega<0$ & arbitrary & arbitrary & $c_{10}<0$ & AS & & & US & US \\
\hline & & & $c_{10}>0$ & US & & & US & US \\
\hline \multirow{5}{*}{$\Omega>0$} & $c_{6}>0$ & $c_{7}>0$ & $c_{10}<0$ & AS & US & US & US & US \\
\cline { 2 - 9 } & $c_{6}>0$ & $c_{7}>0$ & $c_{10}>0$ & US & US & AS & US & US \\
\cline { 2 - 9 } & $c_{6}>0$ & $c_{7}<0$ & $c_{10}<0$ & AS & US & US & US & US \\
\cline { 2 - 9 } & $c_{6}>0$ & $c_{7}<0$ & $c_{10}>0$ & US & AS & US & US & US \\
\cline { 2 - 9 } & $c_{6}<0$ & $c_{7}>0$ & $c_{10}<0$ & AS & US & US & US & US \\
\cline { 2 - 9 } & $c_{6}<0$ & $c_{7}>0$ & $c_{10}>0$ & US & US & AS & US & US \\
\cline { 2 - 9 } & $c_{6}<0$ & $c_{7}<0$ & $c_{10}<0$ & AS & US & US & US & US \\
\cline { 2 - 9 } & $c_{6}<0$ & $c_{7}<0$ & $c_{10}>0$ & US & AS & US & US & US \\
\hline \multirow{2}{*}{$\Omega=0$} & arbitrary & $c_{7} \neq 0$ & $c_{10}<0$ & AS & & & US & US \\
\cline { 2 - 9 } & arbitrary & $c_{7} \neq 0$ & $c_{10}>0$ & US & & & US & US \\
\hline
\end{tabular}

\section{Discussion}

In this paper, we have discussed the reduction of the four types of nonlinear PDEs which are known to have many physically significant applications in physics and related sciences, under four types of GCSs. The resulting equations include a considerable number of nonlinear PDEs which have been widely studied by many authors as their special examples. The exact solutions of these equations can be determined by the compatibility of the GCSs and the considered equations, which reduce to solving systems of nonlinear ODEs. Though the general solution of these systems of ODEs are difficult to obtain, we get many interesting particular solutions. Moreover, these systems of ODEs play an important role in discussing the asymptotic behaviour of the exact solutions such as blow-up property. To illustrate that the approach has wide applications, we derived the exact solutions of additional further examples by the current approach, some of which have been considered by several authors. Our approach strongly provides a symmetry group interpretation for known results. It would be of interest to set up the relationship between the iteration of the nonclassical method [22] and the current approach and give a GCS group interpretation for known results [16] of the multidimensional diffusion equations.

\section{Acknowledgment}

The author would like to thank Dr Liu Q. M. for helpful discussions and the referee for many valuable suggestions, as well as Professors P. A. Clarkson, M. C. Nucci, J. 
R. King and Dr R. Z. Zhdanov for sending me their preprints.

\section{Appendix A}

In this appendix, we present the exact solutions and reductions to systems of ODEs for (2)-(4).

A.1. $u_{t t}=g\left(u, u_{x}\right) u_{x x}+f\left(u, u_{x}\right)$. Analogously to the procedure for case (4.1), we can easily obtain the corresponding results for (2), which are listed as follows.

A.1.1. Under $\sigma_{1}=u_{x x x}+u_{x}$. The exact solution of (2) with (16) is determined by (26), where $\alpha, \beta$ and $\gamma$ satisfy

$$
\alpha_{t t}=L_{1}(\alpha, \beta, \gamma), \quad \beta_{t t}=L_{2}(\alpha, \beta, \gamma), \quad \gamma_{t t}=L_{3}(\alpha, \beta, \gamma),
$$

where $L_{i}, i=1,2,3$ are as in (28).

A.1.2. Under $\sigma_{2}=u_{x x x}-u_{x}$. In this subcase, the exact solution of (2) with (19) is given by (29), where $\alpha, \beta$ and $\gamma$ satisfy

$$
\alpha_{t t}=L_{4}(\alpha, \beta, \gamma), \quad \beta_{t t}=L_{5}(\alpha, \beta, \gamma), \quad \gamma_{t t}=L_{6}(\alpha, \beta, \gamma),
$$

where $L_{4}, L_{5}$ and $L_{6}$ are given by (31).

A.1.3. Under $\sigma_{3}=u_{x x x x}$. The ansatz for the exact solution of (2) with (22) is (32), where $\alpha, \beta, \gamma$ and $\delta$ satisfy

$$
\alpha_{t t}=L_{7}(\alpha, \beta, \gamma), \quad \beta_{t t}=L_{8}(\alpha, \beta, \gamma), \quad \gamma_{t t}=L_{9}(\alpha, \beta, \gamma), \quad \delta_{t t}=18 c_{1} \delta^{2}+c_{4} \delta
$$

A.2. Under $\sigma_{4}=u_{x x x}$. The equation (2) with (25) in this subcase has exact solution given by (35), where $\alpha, \beta$ and $\gamma$ satisfy

$$
\alpha_{t t}=L_{10}(\alpha, \beta, \gamma), \quad \beta_{t t}=L_{11}(\alpha, \beta, \gamma), \quad \gamma_{t t}=L_{12}(\alpha, \beta, \gamma),
$$

where $L_{10}, L_{11}$ and $L_{12}$ are given in (37).

A.3. $u_{t}=-u_{x x x x}+g\left(u, u_{x}\right) u_{x x}+f\left(u, u_{x}\right)$. Equation (3) is a model frequently encountered in the study of continuous media which exhibits a chaotic behaviour [19], including the well-known Kuramoto-Sivashinsky equation as its special case. The GCSs $\sigma_{3}$ and $\sigma_{4}$ imply $u_{x x x x}=0$. Then equation (3) reduces to equation (1). We consider two cases. 
A.4. Under $\sigma_{1}=u_{x x x}+u_{x}$. The ansatz for the exact solution of (3) with constraints (16) also is determined by (26), where $\alpha, \beta$ and $\gamma$ satisfy

$$
\alpha_{t}=L_{1}(\alpha, \beta, \gamma), \quad \beta_{t}=L_{2}(\alpha, \beta, \gamma)-\beta, \quad \gamma_{t}=L_{3}(\alpha, \beta, \gamma)-\gamma,
$$

with $L_{1}, L_{2}$ and $L_{3}$ given by (28).

A.4.1. Under $\sigma_{2}=u_{x x x}-u_{x}$. It is easy to show that $u(t, x)$ takes the form (29). Substitution of (29) into (3) with (19) gives rise to

$$
\alpha_{t}=L_{4}(\alpha, \beta, \gamma), \quad \beta_{t}=L_{5}(\alpha, \beta, \gamma)-\beta, \quad \gamma_{t}=L_{6}(\alpha, \beta, \gamma)-\gamma,
$$

where $L_{4}, L_{5}$ and $L_{6}$ are given by (31).

A.5. $u_{x x x x}+2 u_{x x y y}+u_{y y y y}=g\left(u, u_{x}\right) u_{x x}+f\left(u, u_{x}\right)$. In terms of the GCSs of (4), four subcases arise.

A.5.1. Under $\sigma_{1}=u_{x x x}+u_{x}$. In this subcase, $f$ and $g$ are determined by (16). Integrating $\sigma_{1}=0$ gives

$$
u(x, y)=\alpha(y)+\beta(y) \cos x+\gamma(y) \sin x .
$$

Substitution of (A.7) into (4) with (16) implies $\alpha, \beta$ and $\gamma$ satisfy

$$
\begin{gathered}
\alpha_{y y y y}=L_{1}(\alpha, \beta, \gamma), \quad \beta_{y y y y}-2 \beta_{y y}+\beta=L_{2}(\alpha, \beta, \gamma), \\
\gamma_{y y y y}-2 \gamma_{y y}+\gamma=L_{3}(\alpha, \beta, \gamma),
\end{gathered}
$$

where $L_{1}, L_{2}$ and $L_{3}$ are given in (28).

A.5.2. Under $\sigma_{2}=u_{x x x}-u_{x}$. The ansatz of the exact solution of (4) with (19) is

$$
u(x, y)=\alpha(y)+\beta(y) \cosh x+\gamma(y) \sinh x,
$$

which is compatible with the governing equation (4) and leads to

$$
\begin{gathered}
\alpha_{y y y y}=L_{4}(\alpha, \beta, \gamma), \quad \beta_{y y y y}+2 \beta_{y y}+\beta=L_{5}(\alpha, \beta, \gamma), \\
\gamma_{y y y y}+2 \gamma_{y y}+\gamma=L_{6}(\alpha, \beta, \gamma),
\end{gathered}
$$

where $L_{4}, L_{5}$ and $L_{6}$ are given by (31).

A.5.3. Under $\sigma_{3}=u_{x x x x}$. Solving $\sigma_{3}=0$, we obtain the exact solution of (4) with (22) as

$$
u(x, y)=\alpha(y)+\beta(y) x+\gamma(y) x^{2}+\delta(y) x^{3} .
$$

Substitution of (A.11) into equation (4) yields

$$
\begin{gathered}
\alpha_{y y y y}+4 \gamma_{y y}=L_{7}(\alpha, \beta, \gamma), \quad \beta_{y y y y}+12 \delta_{y}=L_{8}(\alpha, \beta, \gamma), \\
\gamma_{y y y y}=L_{9}(\alpha, \beta, \gamma), \quad \delta_{y y y y}=18 \delta^{2}
\end{gathered}
$$

where $L_{7}, L_{8}$ and $L_{9}$ take the form (34). 
A.5.4. Under $\sigma_{4}=u_{x x x}$. Here the exact solution of (4) with (25) is

$$
u(x, y)=\alpha(y)+\beta(y) x+\gamma(y) x^{2},
$$

and $\alpha, \beta$ and $\gamma$ satisfy

$$
\alpha_{y y y y}+4 \gamma_{y y}=L_{10}(\alpha, \beta, \gamma), \quad \beta_{y y y y}=L_{11}(\alpha, \beta, \gamma), \quad \gamma_{y y y y}=L_{12}(\alpha, \beta, \gamma),
$$

with $L_{10}, L_{11}$ and $L_{12}$ determined by (37).

\section{Appendix B}

In this appendix, we present general solution to a special Abel equation

$$
\alpha_{t}=A \alpha^{3}+B \alpha^{2}+C \alpha+D,
$$

where $A, B, C$ and $D$ are arbitrary constants, which appears in this paper several times. There are three cases to consider.

B.1. $A \neq 0$. In terms of the roots of

$$
A \alpha^{3}+B \alpha^{2}+C \alpha+D=0,
$$

we distinguish four subcases.

B.1.1. There are three distinct real roots. The general solution is

$$
\left(\alpha-a_{1}\right)^{a_{2}-a_{3}}\left(\alpha-a_{2}\right)^{a_{3}-a_{1}}\left(\alpha-a_{3}\right)^{a_{1}-a_{2}}=D_{1} e^{A\left(a_{1}-a_{2}\right)\left(a_{2}-a_{3}\right)\left(a_{1}-a_{3}\right)\left(t-t_{0}\right)},
$$

where $a_{i}, i=1,2,3$, are the three real roots.

B.1.2. There is one real and two conjugate complex roots. In this subcase, we have

$$
A \alpha^{3}+B \alpha^{2}+C \alpha+D=A\left(\alpha-a_{1}\right)\left[\left(\alpha+a_{2}\right)^{2}+a_{3}^{2}\right], \quad a_{3} \neq 0
$$

The general solution of (B.1) is

$$
\frac{1}{\left(a_{1}+a_{2}\right)^{2}+a_{3}^{2}} \ln \frac{\alpha-a_{1}}{\sqrt{\left(\alpha+a_{2}\right)^{2}+a_{3}^{2}}}-\frac{a_{1}+a_{2}}{a_{3}} \arctan \frac{\alpha+a_{2}}{a_{3}}=A\left(t-t_{0}\right) .
$$

B.1.3. There are two distinct, real roots $a_{1}$ and $a_{2}$, the multiplicity of $a_{2}$ being two. So we have for the general solution of (B.1)

$$
\frac{\alpha-a_{2}}{\alpha-a_{1}} e^{\left(a_{2}-a_{1}\right) /\left(\alpha-a_{1}\right)}=e^{A\left(a_{2}-a_{1}\right)^{2}\left(t-t_{0}\right)} .
$$


B.1.4. There is only one real root $a_{1}$, of multiplicity three.

$$
\alpha=a_{1}+\left[2 A\left(t_{0}-t\right)\right]^{-1 / 2} .
$$

B.2. $A=0, B \neq 0$. The equation reduces to the constant Riccati equation and the general solution is given according to three subcases.

B.2.1. $\Delta=C^{2}-4 B D>0$.

$$
\alpha=\frac{a_{1}+a_{2}}{2}+\frac{a_{2}-a_{1}}{2} \operatorname{coth} \frac{\left(a_{1}-a_{2}\right) B\left(t-t_{0}\right)}{2},
$$

where $a_{1}$ and $a_{2}$ are the real roots of (B.2) with $A=0$.

B.2.2. $\Delta<0$.

The general solution of (B.1) is

$$
\alpha=-\frac{C}{2 B}+\frac{\sqrt{4 B D-C^{2}}}{2 B} \tan B\left(t-t_{0}\right) .
$$

B.2.3. $\Delta=0$.

$$
\alpha=-\frac{C}{2 B}-\frac{1}{B\left(t-t_{0}\right)},
$$

which blows up at $t_{0}$.

B.3. $A=B=0, C \neq 0$.

$$
\alpha=e^{C\left(t-t_{0}\right)}-\frac{D}{C}
$$

B.4. $A=B=C=0$.

$$
\alpha=D\left(t-t_{0}\right)
$$

\section{Appendix C}

In this appendix, we derive the general solution to the ordinary differential equation

$$
\alpha_{t}=A \alpha^{4}+B \alpha^{3}+C \alpha^{2}+C \alpha+D .
$$

where $A, B, C$ and $D$ are constants. The case $A=0$ has been considered in Appendix $\mathrm{B}$, so we consider the case $A \neq 0$. Analogously to the discussion for (B.1), in view of the roots of

$$
Q(\alpha)=A \alpha^{4}+B \alpha^{3}+C \alpha^{2}+C \alpha+D=0
$$

we distinguish nine cases. 
C.1. There are four distinct real roots. In this case, we obtain the general solution of (C.1) as

$$
\begin{aligned}
& \left(\alpha-a_{1}\right)^{\frac{1}{\left(a_{2}-a_{1}\right)\left(a_{3}-a_{1}\right)\left(a_{4}-a_{1}\right)}}\left(\alpha-a_{2}\right)^{\frac{1}{\left(a_{1}-a_{2}\right)\left(a_{3}-a_{2}\right)\left(a_{4}-a_{2}\right)}}\left(\alpha-a_{3}\right)^{\frac{1}{\left(a_{1}-a_{3}\right)\left(a_{2}-a_{3}\right)\left(a_{4}-a_{3}\right)}} \\
& \quad \times\left(\alpha-a_{4}\right)^{\frac{1}{\left(a_{1}-a_{4}\right)\left(a_{2}-a_{4}\right)\left(a_{3}-a_{4}\right)}}=e^{A\left(t-t_{0}\right)},
\end{aligned}
$$

where $a_{i}, i=1,2,3,4$ are the different roots of (C.2).

C.2. There are three distinct real roots $a_{i}, i=1,2,3, a_{1}$ having multiplicity two. The general solution of (C.1) in this case is

$$
\begin{gathered}
\ln \left[\left(\alpha-a_{1}\right)^{\frac{a_{2}+a_{3}-2 a_{1}}{\left(a_{2}-a_{1}\right)^{2}\left(a_{3}-a_{1}\right)^{2}}}\left(\alpha-a_{2}\right)^{\frac{1}{\left(a_{2}-a_{3}\left(a_{2}-a_{1}\right)^{2}\right.}}\left(\alpha-a_{3}\right)^{\frac{1}{\left(a_{3}-a_{2}\right)\left(a_{3}-a_{1}\right)^{2}}}\right] \\
-\frac{1}{\left(a_{1}-a_{2}\right)\left(a_{1}-a_{3}\right)\left(\alpha-a_{1}\right)}=A\left(t-t_{0}\right) .
\end{gathered}
$$

C.3. There are two distinct real roots $a_{i}, i=1,2$, both with multiplicity two. We obtain the general solution of (C.1) as

$$
\frac{\alpha-a_{2}}{\alpha-a_{1}}-\frac{\alpha-a_{1}}{\alpha-a_{2}}-2 \ln \frac{\alpha-a_{2}}{\alpha-a_{1}}=A\left(a_{2}-a_{1}\right)^{3}\left(t-t_{0}\right) .
$$

C.4. There are two distinct real roots, $a_{1}$ of multiplicity three and $a_{2}$ of multiplicity one.

$$
\ln \frac{\alpha-a_{1}}{\alpha-a_{2}}+\frac{a_{1}-a_{2}}{\alpha-a_{1}}-\frac{\left(a_{1}-a_{2}\right)^{2}}{2\left(\alpha-a_{1}\right)^{2}}=A\left(a_{1}-a_{2}\right)^{3}\left(t-t_{0}\right)
$$

gives the general solution of (C.1) in this case.

C.5. There is only one real root $a_{1}$, of multiplicity four. The general solution of (C.1) in this case is

$$
\alpha=a_{1}+\left[-\frac{1}{3 A\left(t-t_{0}\right)}\right]^{1 / 3},
$$

which blows up at $t_{0}$.

C.6. There are four distinct complex roots. In this case, we first have

$$
Q(\alpha)=\left[\left(\alpha+a_{1}\right)^{2}+b_{1}^{2}\right]\left[\left(\alpha+a_{2}\right)^{2}+b_{2}^{2}\right], \quad b_{1}, b_{2} \neq 0 .
$$

Hence we obtain the general solution to (C.1) as

$$
\begin{aligned}
\left(a_{1}-\right. & \left.a_{2}\right) \ln \left[\frac{\left(\alpha+a_{1}\right)^{2}+b_{1}^{2}}{\left(\alpha+a_{2}\right)^{2}+b_{2}^{2}}\right]+\left[\left(a_{1}-a_{2}\right)^{2}+b_{2}^{2}-b_{1}^{2}\right] \arctan \frac{\alpha+a_{1}}{b_{1}} \\
& +\left[\left(a_{1}-a_{2}\right)^{2}+b_{1}^{2}-b_{2}^{2}\right] \arctan \frac{\alpha+a_{2}}{b_{2}} \\
= & A\left[\left(a_{1}-a_{2}\right)^{2}+\left(b_{2}-b_{1}\right)^{2}\right]\left[\left(a_{1}-a_{2}\right)^{2}+\left(b_{2}+b_{1}\right)^{2}\right]\left(t-t_{0}\right) .
\end{aligned}
$$


C.7. There are two distinct complex roots of multiplicity two. In this case

$$
Q(\alpha)=\left[\left(\alpha+a_{1}\right)^{2}+b_{1}^{2}\right]^{2} .
$$

The general solution of equation (C.1) is

$$
\frac{b_{1}\left(\alpha+a_{1}\right)}{\left(\alpha+a_{1}\right)^{2}+b_{1}^{2}}+\arctan \frac{\alpha+a_{1}}{b_{1}}=2 A b_{1}^{3}\left(t-t_{0}\right)
$$

C.8. There are one real root of multiplicity two, and two conjugate complex roots. In this case,

$$
Q(\alpha)=\left(\alpha-a_{1}\right)^{2}\left[\left(\alpha+b_{1}\right)^{2}+c_{1}^{2}\right] .
$$

Hence we obtain the general solution of (C.1) as

$$
\begin{gathered}
\left(a_{1}+b_{1}\right) \ln \frac{\left(\alpha+b_{1}\right)^{2}+c_{1}^{2}}{\alpha-a_{1}}+\left[2\left(a_{1}+b_{1}\right)^{2}-1\right] \arctan \frac{\alpha+b_{1}}{c_{1}} \\
-\frac{1}{\alpha-a_{1}}=A\left[\left(a_{1}+b_{1}\right)^{2}+c_{1}^{2}\right]\left(t-t_{0}\right) .
\end{gathered}
$$

C.9. There are two distinct real roots and two conjugate complex roots. We can write

$$
Q(\alpha)=\left(\alpha-a_{1}\right)\left(\alpha-a_{2}\right)\left[\left(\alpha+b_{1}\right)^{2}+c_{1}^{2}\right], \quad c_{1} \neq 0 .
$$

The general solution of (C.1) is then given by

$$
\begin{aligned}
& {\left[\left(a_{1}+b_{1}\right)^{2}+c_{1}^{2}\right] \ln \left(\alpha-a_{2}\right)-\left[\left(a_{2}+b_{1}\right)^{2}+c_{1}^{2}\right] \ln \left(\alpha-a_{1}\right) } \\
& \quad+\frac{\left(a_{2}-a_{1}\right)\left(a_{1}+a_{2}+2 b_{1}\right)}{2} \ln \left[\left(\alpha+b_{1}\right)^{2}+c_{1}^{2}\right] \\
& \quad+\frac{\left(a_{2}-a_{1}\right)\left[\left(b_{1}+c_{1}\right)\left(a_{1}+b_{1}\right)-c_{1}^{2}\right]}{c_{1}} \arctan \frac{\alpha+b_{1}}{c_{1}} \\
&= A\left(a_{2}-a_{1}\right)\left[\left(a_{1}+b_{1}\right)^{2}+c_{1}^{2}\right]\left[\left(a_{2}+b_{1}\right)^{2}+c_{1}^{2}\right]\left(t-t_{0}\right) .
\end{aligned}
$$

\section{References}

[1] K. A. Ames and W. F. Ames, "On group analysis of the Von Karman equations", Nonlin. Anal. 6 (1982) 845-853.

[2] W. F. Ames, R. J. Lohner and E. Adams, "Group properties of $u_{t t}=\left[f(u) u_{x}\right]_{x}$ ", Int. J. Nonlin. Mech. 16 (1981) 439-447. 
[3] D. J. Arrigo and J. M. Hill, "Nonclassical symmetries for nonlinear diffusion and absorption", Stud. Appl. Math. 94 (1995) 21-39.

[4] D. J. Arrigo, J. M. Hill and P. Broadbridge, "Nonclassical symmetry reduction of the linear diffusion equation with a nonlinear source", IMA J. Appl. Math. 52 (1994) 1-24.

[5] W. Bluman and J. D. Cole, "The general similarity solution of the heat equation", J. Math. Mech. 10 (1969) 1025-1042.

[6] P. A. Clarkson and M. D. Kruskal, "New similarity reduction of the Boussinesq equation", J. Math. Phys. 30 (1989) 2201-2213.

[7] P. A. Clarkson and E. M. Mansfield, "Symmetry reductions and exact solutions of a class of nonlinear heat equations", Physica D 70 (1993) 250-288.

[8] V. A. Dordnitsyn, "On invariant solutions of the equation of nonlinear heat conduction with a source", Comp. Math. Phys. 22 (1982) 115-122.

[9] A. S. Fokas and Q. M. Liu, "Generalized conditional symmetries and exact solutions of nonintegrable equations", Theor. Math. Phys. 99 (1994) 263-277.

[10] A.S. Fokas and Q. M. Liu, "Nonlinear interaction of travelling waves of nonintegrable equations", Phys. Rev. Lett. 72 (1994) 3293-3296.

[11] A. S. Fokas and Y. C. Yortsos, "On the exactly solvable equation $S_{t}=\left[(\beta S+\gamma)^{-2} S_{x}\right]_{x}+\alpha(\beta S+$ $\gamma)^{-2} S_{x}$ occurring in two-phase flow in porous media", SIAM J. Appl. Math. 42 (1982) 318-332.

[12] W. Fushchych and R. Zhdanov, "Antireduction and exact solutions of nonlinear heat equations", Nonlin. Math.Phys. 1 (1994) 60-64.

[13] V. A. Galaktionov, "On new exact blow-up solutions for nonlinear heat conduction equations", Diff. Int. Eqns. 3 (1990) 863-874.

[14] W. Hirsch and S. Smale, Differential equations, dynamical systems and linear algebra (Academic, New York, 1974).

[15] N. H. Ibragimov, M. Torrisi and A. Valenti, "Preliminary group classification of equation $V_{t}=$ $f\left(V, V_{x}\right) V_{x x}+g\left(x, V_{x}\right) "$, J. Math. Phys. 32 (1991) 2988-2995.

[16] J. R. King, "Exact polynomial solutions to some nonlinear diffusion equations", Physica D 64 (1993) 35-65.

[17] S. Lie, "Über die Integration dürch bestimmte Integrals von einer Klasse linearer partiller Differentialgleichungen", Arch. Math. 6 (1881) 328-368.

[18] Q. M. Liu and A. S. Fokas, "Exact solutions of solitary wave for certain nonintegrable equation", J. Math. Phys. 37 (1996) 324-345.

[19] P. Manneville, The Kuramoto-Sivashinsky equation: a propagation in systems far from equilibrium (Springer, Berlin, 1988).

[20] J. D. Murray, Mathematical Biology (Springer, Berlin, 1989).

[21] A. K. Myers-Beghton and D. D. Vvdensky, "Nonlinear equation for diffusion and adatom interaction during epitaxial growth on vininal surfaces", Phys. Rev. B 42 (1990) 5544-5554.

[22] M. C. Nucci, "Iterating the nonclassical symmetries method", Physica D 7 (1994) 124-134.

[23] P. J. Olver and P. Rosenau, "Group invariant solutions of differential equations", SIAM J. Appl. Math. 47 (1987) 263-278.

[24] L. V. Ovsiannikov, Group Analysis of Differential Equations (Academic Press, New York, 1982).

[25] C. Z. Qu, "New exact solutions to $N$-dimensional radially symmetric nonlinear diffusion equation", Int. J. Theor. Phys. 35 (1996) 2679-2687.

[26] C. Z. Qu, "Group classification and generalized conditional symmetry reduction of the nonlinear diffusion-convection equation with a nonlinear source", Stud. Appl. Math. 99 (1997) 107-136.

[27] F. Schwarz, "Lie symmetries of the Von Karman equations", Compt. Phys. Commun. 31 (1984) 113-114.

[28] S. Wiggins, Introduction to applied nonlinear dynamical systems and chaos (Springer-Verlag, Berlin, 1990). 
[29] H. Wilhelmsson, "Explosive instabilities of reaction-diffusion equation", Phys. Rev. A 36 (1987) 965-966.

[30] C. M. Yung, K. Verburg and P. Baveye, "Group classification and symmetry reductions of the nonlinear diffusion-convection equation $u_{t}=\left(D(u) u_{x}\right)_{x}-K^{\prime}(u) u_{x}$ ", Int. J. Nonlin. Mech. 29 (1994) 273-278.

[31] Z. F. Zhang, T. R. Ding, W. Z. Huang and Z. X. Dong, Qualitative Theory of Differential Equations (Science, Beijing, 1985).

[32] R. Z. Zhdanov, "Conditional Lie-Backlund symmetry and reduction of evolution equation", $J$. Phys. A: Math. Gen. 28 (1995) 3841-3850. 